\title{
Ring pattern solutions of a free boundary problem in diblock copolymer morphology
}

\author{
Xiaosong Kang * \\ School of Mathematics and Statistics \\ Wuhan University \\ Wuhan, Hubei 430072, China
}

\author{
Xiaofeng Ren ${ }^{\dagger \ddagger}$ \\ Department of Mathematics \\ The George Washington University \\ Washington, DC 20052, USA
}

January 4, 2009

\begin{abstract}
The cross section of a diblock copolymer in the cylindrical phase is made up of a large number of microdomains of small discs with high concentration of the minority monomers. Often several ring like microdomains appear among the discs. We show that a ring like structure may exist as a stable solution of a free boundary problem derived from the Ohta-Kawasaki theory of diblock copolymers. The existence of such a stable, single ring structure explains why rings exist for a long period of time before they eventually disappear or become discs in a diblock copolymer. A variant of Lyapunov-Schmidt reduction process is carried out that rigorously reduces the free boundary problem to a finite dimensional problem. The finite dimensional problem is solved numerically. A stability criterion on the parameters determines whether the ring solution is stable.
\end{abstract}

Key words. diblock copolymer morphology, ring pattern, defect.

2000 AMS subject classification. 35R35, 82B24, 82D60.

Abbreviated title. Ring pattern solutions.

\section{Introduction}

A molecule in a diblock copolymer is a linear chain of an A-monomer block grafted covalently to a B-monomer block. Because of the repulsion between the unlike monomers, the different type sub-chains tend to segregate, but as they are chemically bonded in chain molecules, segregation of sub-chains cannot lead to a macroscopic phase separation. Only a local micro-phase separation occurs: micro-domains rich in A monomers and micro-domains rich in B monomers emerge as a result. The patterns formed from the micro-domains are known as morphology phases. Various phases, including lamellar, cylindrical, spherical, gyroid, etc, have been observed in experiments. See Bates and Fredrickson [1]. Figure 1 shows the lamellar, cylindrical, and spherical phases.

However it is known that often times a diblock copolymer exists in a defective state (see Tsori, Andelman and Schick [28]). In [28] Chevron- and $\Omega$-shaped tilt boundaries are studied in the lamellar phase of a diblock copolymer. A non-ideal lamellar phase is found in [20] where micro-domains are separated by wiggling interfaces.

*Supported in part by NSF of China grant 10871152.

${ }^{\dagger}$ Corresponding author. Phone: 1202 994-6791; Fax: 1202 994-6760; E-mail: ren@math.usu.edu

¥Supported in part by NSF grants DMS-0509725, DMS-0754066. 

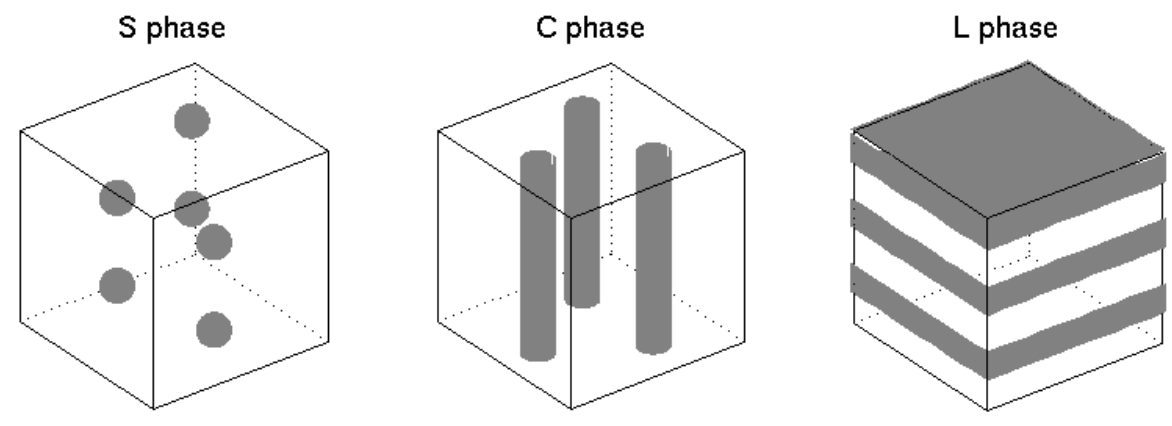

Figure 1: The spherical, cylindrical, and lamellar morphology phases commonly observed in diblock copolymer melts. The dark color indicates the concentration of type A monomers, and the white color indicates the concentration of type B monomers.

In this paper we study a type of defect in the cylindrical phase of a diblock copolymer. In $[24,23]$ Ren and Wei studied a cross section of an ideal cylindrical phase which consists of a number of circular discs of approximately equal size. However before one finds discs, numerical simulations with the Ohta-Kawasaki density functional theory often show the existence of rings among discs. Figure 2 shows snap shots of the Otha-Kawasaki functional [12] under its gradient flow. We observe four rings among many discs. They exist for a very long period of time in the flow, before they eventually disappear or change to discs. Therefore we believe that a single ring, by its self, may be a stable structure. Only through interaction with other discs, a ring becomes unstable. This type of instability appears to be fairly weak, allowing rings to exist for a long time and easily observable.

We will verify the observation that a single ring structure may exist as a local minimum of the free energy functional, and hence a stable solution to the Euler-Lagrange equation of the free energy.

By a ring we mean a set like $\left\{x \in R^{2}: r_{1}<|x-\xi|<r_{2}\right\}$ in $R^{2}$ where $\xi$ is the center and $r_{1}$ and $r_{2}$ the inner and outer radii of the ring respectively. As a solution to a free boundary problem (1.1) given later, a ring structure actually deviates slightly from the set described above. The inner and outer boundaries of a ring solution differ a bit from perfect circles. The exact amount of deviation and the center $\xi$ and the radii $r_{1}$ and $r_{2}$ will all be determined as we solve (1.1).

Let a cross section of a diblock copolymer sample be $D$. Assume that $D$ is a bounded and sufficiently smooth domain in $R^{2}$. Suppose that the system is in a strongly segregated state and the A-monomers and the B-monomers are locally separated. Let A-monomers occupy the subset $E$ and the B-Monomers occupy the subset $D \backslash E$. Denote the Lebesgue measure of $E$ by $|E|$ and denote the part of the boundary of $E$ that is in $D$ by $\partial_{D} E$. Let $\chi_{E}$ be the characteristic function of $E$, i.e. $\chi_{E}(x)=1$ if $x \in E$, and $\chi_{E}(x)=0$ if $x \in D \backslash E$. Given a fixed number $a \in(0,1)$ we look for a subset $E$ of $D$ and a number $\lambda$ such that $\partial_{D} E$ is a smooth curve, or a union of several smooth curves, $|E|=a|D|$, and at every point on $\partial_{D} E$

$$
H\left(\partial_{D} E\right)+\gamma(-\Delta)^{-1}\left(\chi_{E}-a\right)=\lambda .
$$

Here $H\left(\partial_{D} E\right)$ is the curvature of $\partial_{D} E$ viewed from $E$ and $\gamma$ is a given positive number. The expression $(-\Delta)^{-1}\left(\chi_{E}-a\right)$ is the solution $v$ of the problem

$$
-\Delta v=\chi_{E}-a \text { in } D, \partial_{\nu} v=0 \text { on the boundary of } D, \quad \bar{v}=0
$$

where the bar over a function is the average of the function over its domain, e.g.

$$
\bar{v}=\frac{1}{|D|} \int_{D} v(x) d x .
$$



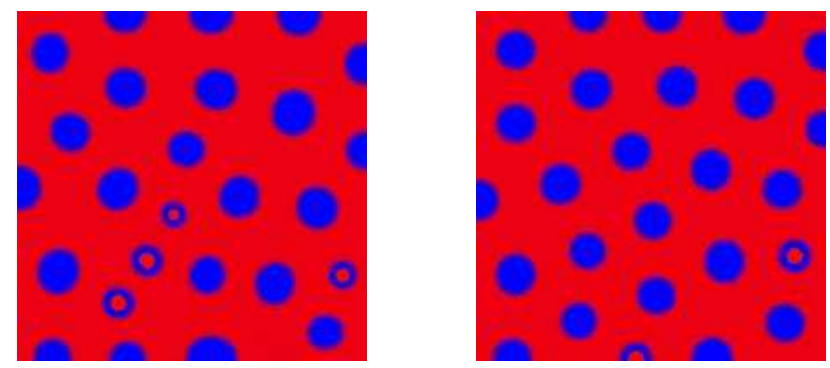

Figure 2: Rings among discs, found from a numerical simulation of the Ohta-Kawasaki model. The governing equation is $u_{t}=\Delta\left(-\epsilon^{2} \Delta u+u(u-1 / 2)(u-1)\right)-\epsilon \gamma(u-a)$ with zero Neumann boundary condition for $u$ and $\Delta u$. The sample size is 20 by $20, \epsilon=0.1, \gamma=1, a=0.225$. The grid size is 0.1 and the time step is 0.002. A finite difference method with the implicit scheme for the highest order term and the explicit scheme for the lower order terms is used. Initially $u(x, 0)$ is a small perturbation of $a$. Four rings are observed at $t=100$; at $t=5000$ only two are left.

Because $(-\Delta)^{-1}$ is a nonlocal operator, the free boundary problem (1.1) is nonlocal.

The equation (1.1) is the Euler-Lagrange equation of the free energy functional of the system. The functional takes the form

$$
J(E)=\left|D \chi_{E}\right|(D)+\frac{\gamma}{2} \int_{D}\left|(-\Delta)^{-1 / 2}\left(\chi_{E}-a\right)\right|^{2} d x
$$

The admissible set $\Sigma$ of the functional $J$ is the collection of all measurable subsets of $D$ of measure $a|D|$ and of finite perimeter, i.e.

$$
\Sigma=\left\{E \subset D: E \text { is Lebesgue measurable, }|E|=a|D|, \quad \chi_{E} \in B V(D)\right\} .
$$

Here $B V(D)$ is the space of functions of bounded variation on $D$. The nonlocal integral operator $(-\Delta)^{-1}$ is defined by solving

$$
-\Delta v=q \text { in } D, \partial_{\nu} v=0 \text { on the boundary of } D, \bar{v}=0
$$

for $q \in L^{2}(D), \bar{q}=0$. Then $(-\Delta)^{-1 / 2}$ is the positive square root of $(-\Delta)^{-1}$.

Since $\chi_{E} \in B V(D)$, we view $D \chi_{E}$, the derivative of $\chi_{E}$, as a vector valued, signed measure, and let $\left|D \chi_{E}\right|$ be the positive total variation measure of $D \chi_{E}$. The first term in $(1.2),\left|D \chi_{E}\right|(D)$, is the $\left|D \chi_{E}\right|$ measure of the entire domain $D$, which is known as the perimeter of $E$. When $\partial_{D} E$ is a smooth curve, or a union of smooth curves, $\left|D \chi_{E}\right|(D)$ is just the length of $\partial_{D} E$. For this reason $\left|D \chi_{E}\right|(D)$ is called the perimeter of $E$ in $D$ and sometimes denoted by $P_{D}(E)$. See [7, Section 5.7] for more information on $P_{D}(E)$. The constant $\lambda$ in (1.1) comes as a Lagrange multiplier from the constraint $|E|=a|D|$.

The functional $J$ in (1.2) is derived from the Ohta-Kawasaki density functional theory of diblock copolymers as the strong segregation limit at low temperature. Mathematically $J$ is the $\Gamma$-limit of the Ohta-Kawasaki free energy (Ren and Wei [13]). The A-monomers occupy the set $E$ and the B-monomers occupy the set $D \backslash E$. The number $a$ is the block composition fraction. It is the number of the A-monomers divided by the number of all the A- and B- monomers in a polymer chain. The interface between the A-monomer regions and $\mathrm{B}$-monomer regions is $\partial_{D} E$ whose tension is its length. The connectivity of $\mathrm{A}$ and $\mathrm{B}$ monomers in a chain molecule is described by the nonlocal term in $J$.

Nishiura and Ohnishi [10] formulated the Ohta-Kawasaki theory on a bounded domain as a singularly perturbed variational problem with a nonlocal term. They also formally identified the free boundary problem (1.1). Since then much work has been done to these problems. The lamellar phase was studied by Ren and Wei [13, 15, 16, 20, 21], Fife and Hilhorst [8], Chen and Oshita [2], 
and Choksi and Sternberg [6]. The work of Müller [9] was related to the lamellar phase in the case $a=1 / 2$, as observed in [10]. Radially symmetric bubble and ring patterns were studied by Ren and Wei $[14,19,22]$. The cylindrical phase and the spherical phase were studied by Ren and Wei $[24,23,25]$. A triblock copolymer in the lamellar phase was studied by Ren and Wei [18]. Teramoto and Nishiura [26] studied the gyroid phase numerically. Mathematically strict derivations of the density functional theories for diblock copolymers, triblock copolymers and polymer blends were given by Choksi and Ren [4, 5], and Ren and Wei [17]. Also see Ohnishi and Nishiura [11], Ohnishi et al [11], and Choksi [3].

The main result of this paper, Theorem 2.1, is a variant of Lyapunov-Schmidt reduction from (1.1) to a finite dimensional problem. Whether there is a ring solution depends on whether the finite dimensional problem is solvable. The finite dimensional problem is not too complex, but we still have to study it numerically. We find the existence of a ring solution if $a$ is small and $\gamma$ is in a proper range (see Observation 2.3). In the reduction procedure a stability criterion, Theorem 2.2, is found which determines whether the ring solution is stable. This criterion is part of the finite dimensional problem and is used numerically. It appears that the ring solution is stable if $\gamma$ is large and unstable if $\gamma$ is small (see Observation 2.3).

At the technical level, the difference between the results obtained here and the ones in [23, 25] is that the radius of a disc is to the leading order determined by the constraint $|E|=a|D|$, but a priori the size of a ring, i.e. the inner radius and the outer radius, is not known even approximately. It is found by solving the reduced problem. In the reduction process, a new matrix is discovered (see $M_{n}$ in 5.14) which allows us to analyze the spectrum of the operator in the Fourier space.

\section{Theorems and observations}

Let $R_{1}, R_{2}>0$ be such that

$$
R_{2}^{2}-R_{1}^{2}=1
$$

For each integer $n \geq 2$ the quadratic equation

$$
\frac{1-\left(\frac{R_{1}}{R_{2}}\right)^{2 n}-n\left(1-\left(\frac{R_{1}}{R_{2}}\right)^{2}\right)}{16 n^{2}} \Gamma^{2}+\left[\frac{n^{2}-1}{8 n R_{2}^{3}}+\frac{n^{2}-1}{8 R_{1}^{3}}\left(\frac{1}{n}-1+\left(\frac{R_{1}}{R_{2}}\right)^{2}\right)\right] \Gamma+\frac{\left(n^{2}-1\right)^{2}}{4 R_{1}^{3} R_{2}^{3}}=0
$$

of $\Gamma$ has one positive root and one negative root, because the graph of the left side, as a function of $\Gamma$, is a downward parabola, and when $\Gamma=0$ the left side is positive. Denote the positive root by $\tilde{\Gamma}_{n}\left(R_{1}\right)$ as a quantity that depends on $R_{1}$. Define curves $W_{n}$ in the first quadrant of the $R_{1}-\Gamma$ plane by

$$
W_{n}=\left\{\left(R_{1}, \tilde{\Gamma}_{n}\left(R_{1}\right)\right): R_{1}>0\right\}, n=2,3, \ldots
$$

The $\tilde{\Gamma}_{n}$ 's have the property that for each $n=2,3, \ldots$,

$$
\lim _{R_{1} \rightarrow 0} \tilde{\Gamma}_{n}\left(R_{1}\right)=2 n(n+1) .
$$

For large $R_{1}$ there is the asymptotic formula

$$
\lim _{R_{1} \rightarrow \infty} \frac{\tilde{\Gamma}_{n}\left(R_{1}\right)}{R_{1}}=8(n+1)
$$

for each $n=2,3, \ldots$. Moreover when $R_{1}$ is in a compact subset of $[0, \infty)$,

$$
\lim _{n \rightarrow \infty} \frac{\tilde{\Gamma}_{n}\left(R_{1}\right)}{n^{2}}=\frac{2}{\sqrt{1+R_{1}^{2}}}
$$

uniformly. Here $\tilde{\Gamma}_{n}(0)$ is defined by the limit in $(2.3)$. 
Next we define $Q_{\Gamma}$ as a function of $R_{1}>0$ by

$$
Q_{\Gamma}\left(R_{1}\right)=R_{1}+\sqrt{1+R_{1}^{2}}+\frac{\Gamma}{4}\left[\frac{R_{1}^{2}}{4}\left(R_{1}^{2} \log \frac{1+R_{1}^{2}}{R_{1}^{2}}-1\right)+\frac{1}{4} \log \frac{1}{1+R_{1}^{2}}\right] .
$$

In the function $Q_{\Gamma}, \Gamma$ is a positive parameter.

Let us denote the Green's function of $-\Delta$ by $G$. It is a sum of two parts:

$$
G(x, y)=\frac{1}{2 \pi} \log \frac{1}{|x-y|}+R(x, y) .
$$

The regular part of $G(x, y)$ is $R(x, y)$. The Green's function satisfies the equation

$$
-\Delta_{x} G(x, y)=\delta(x-y)-\frac{1}{|D|} \text { in } D, \quad \partial_{\nu(x)} G(x, y)=0 \text { on } \partial D, \overline{G(\cdot, y)}=0 \forall y \in D
$$

Here $\Delta_{x}$ is the Laplacian with respect to the $x$-variable of $G, \nu(x)$ is the outward normal direction at $x \in \partial D$, and $\partial_{\nu(x)}$ is the normal derivative there with respect to the $x$-variable.

Our first theorem addresses the existence issue.

Theorem 2.1 If there is a point $\left(S_{0,1}, \Gamma\right)$ in the first quadrant of the $R_{1}-\Gamma$ plane such that

1. $\left(S_{0,1}, \Gamma\right) \notin \cup_{n=2}^{\infty} W_{n}$, and

2. $S_{0,1}$ minimizes $Q_{\Gamma}$ locally,

then the Euler-Lagrange equation (1.1) with $\gamma=\Gamma\left(\frac{a|D|}{\pi}\right)^{-3 / 2}$ admits a ring pattern solution, when a is sufficiently small. The inner radius of the ring is approximately $S_{0,1}\left(\frac{a|D|}{\pi}\right)^{1 / 2}$ and the outer radius of the ring is approximately $S_{0,2}\left(\frac{a|D|}{\pi}\right)^{1 / 2}$ where $S_{0,2}^{2}-S_{0,1}^{2}=1$. The center of the ring is close to a minimum of the function $R(x, x), x \in D$.

The next theorem shows when one can have a stable solution.

Theorem 2.2 If the first condition in Theorem 2.1 is satisfied because $\left(S_{0,1}, \Gamma\right)$ is below all the curves $W_{n}$, then the solution constructed in Theorem 2.1 is stable. If the first condition in Theorem 2.1 is satisfied and $\left(S_{0,1}, \Gamma\right)$ lies between two of the $W_{n}$ 's, then the solution is unstable.

These two theorems reduce the existence and the stability of a solution to a finite dimensional problem. We study this finite dimensional problem numerically.

The curves $W_{n}$ are plotted in Figure 3. We see that these curves appear in the increasing order as $n$ gets larger.

The function $Q_{\Gamma}$ admits a positive local minimum only if $\Gamma$ is sufficient large (see Figure 4 ). We have found numerically that there is a constant $\Gamma_{0}>0$ such that if $\Gamma>\Gamma_{0}, Q_{\Gamma}$ has a positive local minimum. For each $\Gamma>\Gamma_{0}$ we denote this positive local minimum by $S_{0,1}(\Gamma)$. The curve $V=\left\{\left(S_{0,1}(\Gamma), \Gamma\right): \Gamma>\Gamma_{0}\right\}$ is also plotted on Figure 3. Note that the curve $V$ intersects the curve $W_{2}$ at one point, which we denote by $\left(S_{0,1}\left(\Gamma_{1}\right), \Gamma_{1}\right)$ where $\Gamma_{1}>\Gamma_{0}$. It does not intersect with the other $W_{n}$ 's, $n=3,4, \ldots$.

Based on these numerical calculations, we have the following observation.

Observation 2.3 There exist two universal constants $\Gamma_{0}$ and $\Gamma_{1}$, with $0<\Gamma_{0}<\Gamma_{1}$, such that for any compact subset $\mathcal{K}$ of $\left(\Gamma_{0}, \Gamma_{1}\right) \cup\left(\Gamma_{1}, \infty\right)$ there is a constant $a_{0}>0$ such that if

$$
a<a_{0} \text { and } \gamma\left(\frac{a|D|}{\pi}\right)^{3 / 2} \in \mathcal{K},
$$

there exists a ring pattern solution of (1.1).

If $\gamma\left(\frac{a|D|}{\pi}\right)^{3 / 2}$ is in $\mathcal{K} \cap\left(\Gamma_{0}, \Gamma_{1}\right)$, then the solution is unstable; if $\gamma\left(\frac{a|D|}{\pi}\right)^{3 / 2}$ is in $\mathcal{K} \cap\left(\Gamma_{1}, \infty\right)$, then the solution is stable. 


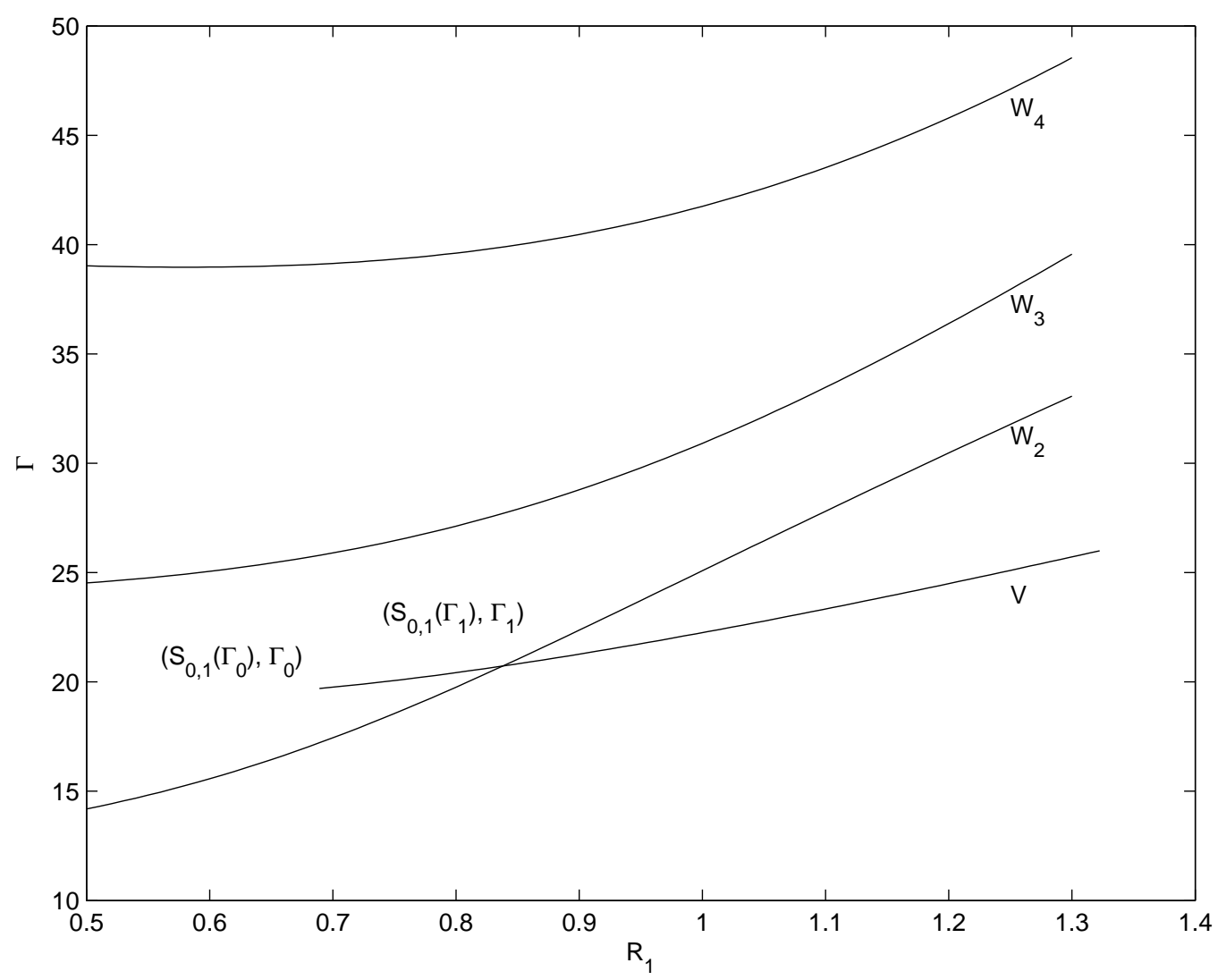

Figure 3: The illustration of $\Gamma_{0}$ and $\Gamma_{1}$.
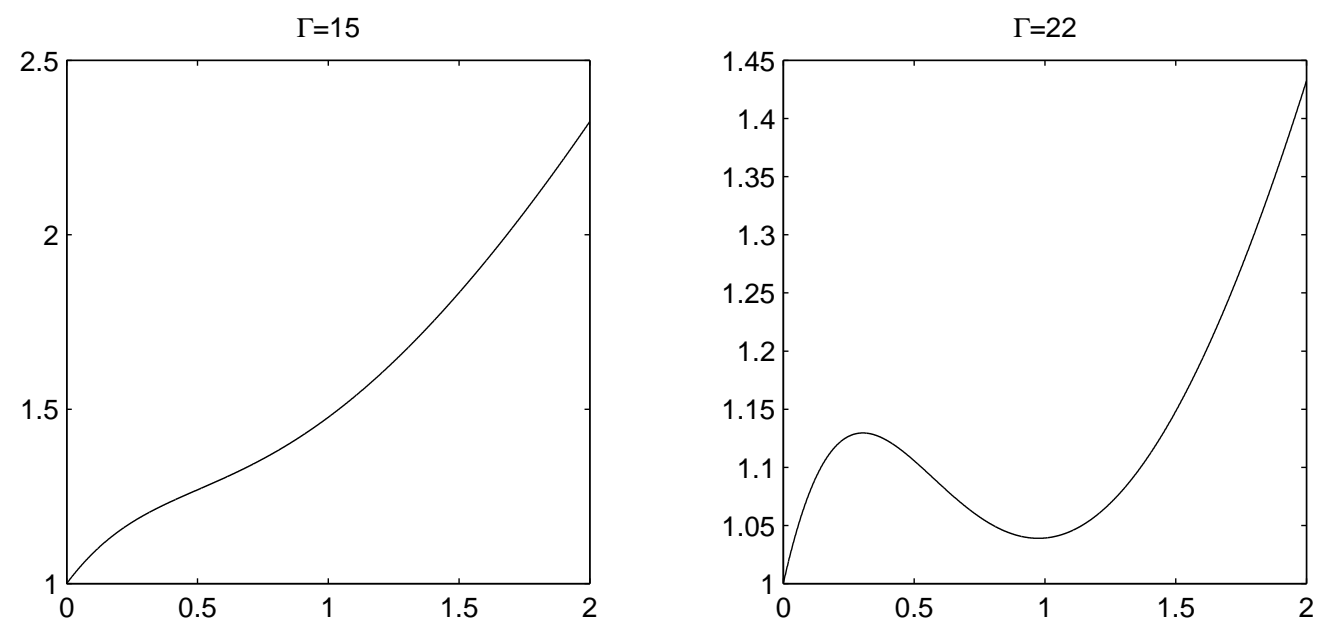

Figure 4: Two graphs of $Q_{\Gamma}$ with $\Gamma=15$ and $\Gamma=22$. The first one does not have a local minimum but the second one does. 
The proofs of the two theorems start with a family of approximate solutions $R=\left\{x \in R^{2}\right.$ : $\left.r_{1}<|x-\xi|<r_{2}\right\}$ which are perfect rings. These approximate solutions are parametrized by the center $\xi$ in $D$ and the inner radius $r_{1}$. The outer radius $r_{2}$ is determined from the inner radius via $\pi r_{2}^{2}-\pi r_{1}^{2}=a|D|$ since $|R|=a|D|$.

Since we look for an exact solution that deviates only a little from one $R$ in the family of the approximate solutions, we perturb each ring $R$ by a pair of $2 \pi$-periodic functions $\phi=\left(\phi_{1}, \phi_{2}\right)$ where $r_{1}^{2}+\phi_{1}$ is the perturbed inner radius square and $r_{2}^{2}+\phi_{2}$ the perturbed outer radius square. The perturbed ring is the set

$$
E_{\phi}=\left\{\xi+\alpha e^{i \theta}: \theta \in[0,2 \pi], \alpha \in\left(\sqrt{r_{1}^{2}+\phi_{1}(\theta)}, \sqrt{r_{2}^{2}+\phi_{2}(\theta)}\right)\right\}
$$

Here we perturbed the radius squares instead of radii so that the constraint $\left|E_{\phi}\right|=a|D|$ becomes a simple linear constraint on $\phi_{1}$ and $\phi_{2}$.

In terms of $\phi$ the Euler-Lagrange equation (1.1) is written as $\mathcal{S}(\phi)=0$ where $\mathcal{S}$ is a two component, nonlinear, integro-differential operator from a function space $\mathcal{X}$ to another function space $\mathcal{Y}$.

There is a subset $\mathcal{X}_{*}$ of $\mathcal{X}$ which, roughly speaking, ignores the effect of the translation of the center $\xi$ and the change of the inner radius $r_{1}$. There is a also a corresponding subset $\mathcal{Y}_{*}$ of $\mathcal{Y}$. Given a pair $\left(\xi, r_{1}\right)$, we look for $\varphi=\varphi\left(\theta, \xi, r_{1}\right)$ in $\mathcal{X}_{*}$ that solves the equation up to translation of $\xi$ and change of $r_{1}$, i.e. $\Pi \mathcal{S}(\varphi)=0$ where $\Pi$ is the projection operator from $\mathcal{Y}$ to $\mathcal{Y}_{*}$.

Finally we study the dependence of $J\left(\varphi\left(\cdot, \xi, r_{1}\right)\right)$ on $\left(\xi, r_{1}\right)$. Under the conditions of Theorem 2.1 there exists $\left(\zeta, s_{1}\right)$ such that $J\left(\varphi\left(\cdot, \xi, r_{1}\right)\right)$ is minimized at $\left(\xi, r_{1}\right)=\left(\zeta, s_{1}\right)$. It turns out that at this minimum, $S\left(\varphi\left(\cdot, \zeta, s_{1}\right)\right)=0$.

Whether the solution $\varphi\left(\cdot, \xi, r_{1}\right)$ of $\Pi \mathcal{S}(\varphi)=0$ at each $\left(\xi, r_{1}\right)$ is a local minimizer of $J$ restricted on $\mathcal{X}_{*}$ is the issue addressed in Theorem 2.2. If so, the solution $\varphi\left(\cdot, \zeta, s_{1}\right)$ of $\mathcal{S}(\varphi)=0$ is interpreted as a stable solution. Otherwise it is considered unstable.

In this paper the interval $[0,2 \pi]$ with 0 and $2 \pi$ identified is denoted by $S^{1}$. The $L^{2}$ space on $S^{1}$ is $L^{2}\left(S^{1}\right)$. The inner product in $L^{2}\left(S^{1}\right)$ is denoted by $\langle\cdot, \cdot\rangle$. The $L^{2}$ norm is denoted by $\|\cdot\|_{L^{2}}$, and the $L^{\infty}$ norm by $\|\cdot\|_{L^{\infty}}$. The Sobolev $W^{k, 2}$ space is denoted by $H^{k}\left(S^{1}\right)$ where $k \geq 1$ is an integer. The $W^{k, 2}$ norm is denoted by $\|\cdot\|_{H^{k}}$.

The constant $C$ denotes a positive number which is independent of $a$. It can only depend on the pair $\left(S_{0,1}, \Gamma\right)$ and the domain $D$. The value of $C$ usually changes from place to place.

The point $(\cos \theta, \sin \theta)$ is often written as $e^{i \theta}$ for a simpler notation even though no complex structure is assumed on $R^{2}$. The reader will see things like $e^{i \theta} \cdot x$ which is simply the inner product of two real vectors $(\cos \theta, \sin \theta)$ and $x$.

From now on let $S_{0,1}$ and $\Gamma$ be two numbers satisfying the two conditions in Theorem 2.1. Assume that

$$
\gamma=\Gamma\left(\frac{a|D|}{\pi}\right)^{-3 / 2}
$$

throughout the rest of the paper.

\section{$3 \quad$ Perfect rings as approximate solutions}

Let $U_{1}$ be a neighborhood of the set

$$
\left\{z \in D: R(z, z)=\min _{x \in D} R(x, x)\right\} .
$$

Since $R(x, x) \rightarrow \infty$ as $x \rightarrow \partial D$, the set defined in (3.1) is compact and we can choose $U_{1}$ so that the closure of $U_{1}$ in $R^{2}$ is compact and is contained in $D$.

Denote by $R$ a perfect ring in $D$ centered at $\xi \in U_{1}$ and of inner radius $r_{1}$ and outer radius $r_{2}$ :

$$
R=\left\{x \in R^{2}: r_{1}<|x-\xi|<r_{2}\right\}
$$


We often write $R=B_{2} \backslash B_{1}$ (up to a set of Lebesgue measure 0) where $B_{k}=\left\{x \in R^{2}:|x-\xi|<r_{k}\right\}$, $k=1,2$. Choose $r_{1}$ to be close to $S_{0,1}\left(\frac{a|D|}{\pi}\right)^{1 / 2}$. Namely we let

$$
r_{1} \in U_{2}=\left(\left(S_{0,1}-\delta_{2}\right)\left(\frac{a|D|}{\pi}\right)^{1 / 2},\left(S_{0,1}+\delta_{2}\right)\left(\frac{a|D|}{\pi}\right)^{1 / 2}\right), \quad r_{2}^{2}-r_{1}^{2}=\frac{a|D|}{\pi} .
$$

In (3.3) $\delta_{2}>0$ is a small number, independent of $a$, so that $S_{0,1}$ minimizes $Q_{\Gamma}$ on $\left(S_{0,1}-\delta_{2}, S_{0,1}+\delta_{2}\right)$. Our constructions of $U_{1}$ and $U_{2}$ guarantee that $R$ is inside $D$ if $a$ is sufficiently small.

We plug $R$ into the left side of the equation (1.1) and see, as an approximate solution, how much error $R$ has. Note that when we read (1.1), the curvature of $\partial R$ is viewed from the set $R$, so on the inner circle the curvature is $-\frac{1}{r_{1}}$ and on the outer circle the curvature is $\frac{1}{r_{2}}$.

Lemma 3.1 If $E=R$, the left side of (1.1) is

$$
-\frac{1}{r_{1}}+\gamma\left[-\frac{r_{2}^{2} \log r_{2}-r_{1}^{2} \log r_{1}}{2}+\frac{a|D|}{4 \pi}+a|D| R(\xi, \xi)+\frac{\pi\left(r_{2}^{4}-r_{1}^{4}\right)}{8|D|}\right]+O(1)
$$

on the inner circle of $R$, and is

$$
\frac{1}{r_{2}}+\gamma\left[-\frac{a|D|}{2 \pi} \log r_{2}+a|D| R(\xi, \xi)+\frac{\pi\left(r_{2}^{4}-r_{1}^{4}\right)}{8|D|}\right]+O(1)
$$

on the outer circle of $R$.

Proof. Let $v=(-\Delta)^{-1}\left(\chi_{R}-a\right)=v_{2}-v_{1}$, where $v_{k}, k=1,2$, satisfies

$$
-\Delta v_{k}=\chi_{B_{k}}-\frac{\pi r_{k}^{2}}{|D|} \text { in } D, \quad \partial_{\nu} v_{k}=0 \text { on } \partial D, \quad \overline{v_{k}}=0
$$

Define

$$
P_{k}(x)=\left\{\begin{array}{ll}
-\frac{|x|^{2}}{4}+\frac{r_{k}^{2}}{4}-\frac{r_{k}^{2}}{2} \log r_{k}, & \text { if }|x|<r_{k} \\
-\frac{r_{k}^{2}}{2} \log |x|, & \text { if }|x| \geq r_{k}
\end{array} .\right.
$$

Then $-\Delta P_{k}(\cdot-\xi)=\chi_{B_{k}}$. Write $v_{k}=P_{k}(\cdot-\xi)+Q_{k}(\cdot, \xi)$. Clearly

$$
-\Delta Q_{k}(x, \xi)=-\frac{\pi r_{k}^{2}}{|D|}, \partial_{\nu} Q_{k}(x, \xi)=\partial_{\nu} \frac{r_{k}^{2}}{2} \log |x-\xi| \text { on } \partial D, \overline{Q_{k}(\cdot, \xi)}=-\overline{P_{k}(|\cdot-\xi|)} .
$$

Here the Laplacian $\Delta$ and the outward normal derivative $\partial_{\nu}$ are taken with respect to $x$.

Note that from $(2.8), Q_{k}(x, \xi)$ and $\pi r_{k}^{2} R(x, \xi)$ satisfy the same equation and the same boundary condition. Therefore they can differ only by a constant. This constant is $\overline{Q_{k}(\cdot, \xi)}-\pi r_{k}^{2} \overline{R(\cdot, \xi)}$. But $\overline{v_{k}}=\overline{G(\cdot, \xi)}=0$ implies that this constant is also equal to

$$
-\frac{r_{k}^{2}}{2} \overline{\log |\cdot-\xi|}-\overline{P_{k}(\cdot-\xi)}=\frac{\pi r_{k}^{4}}{8|D|} .
$$

Hence

$$
Q_{k}(x, \xi)=\pi r_{k}^{2} R(x, \xi)+\frac{\pi r_{k}^{4}}{8|D|}
$$

Therefore, direct calculations show that, at each $\xi+r_{1} e^{i \theta}$, a point on the inner circle,

$$
-\frac{1}{r_{1}}+\gamma v\left(\xi+r_{1} e^{i \theta}\right)
$$




$$
\begin{aligned}
& =-\frac{1}{r_{1}}+\gamma\left[-\frac{r_{2}^{2} \log r_{2}-r_{1}^{2} \log r_{1}}{2}+\frac{r_{2}^{2}-r_{1}^{2}}{4}+\pi\left(r_{2}^{2}-r_{1}^{2}\right) R\left(\xi+r_{1} e^{i \theta}, \xi\right)+\frac{\pi\left(r_{2}^{4}-r_{1}^{4}\right)}{8|D|}\right] \\
& \left.=-\frac{1}{r_{1}}+\gamma\left[-\frac{r_{2}^{2} \log r_{2}-r_{1}^{2} \log r_{1}}{2}+\frac{a|D|}{4 \pi}+a|D| R\left(\xi+r_{1} e^{i \theta}, \xi\right)\right]+\frac{\pi\left(r_{2}^{4}-r_{1}^{4}\right)}{8|D|}\right] \\
& =-\frac{1}{r_{1}}+\gamma\left[-\frac{r_{2}^{2} \log r_{2}-r_{1}^{2} \log r_{1}}{2}+\frac{a|D|}{4 \pi}+a|D| R(\xi, \xi)+\frac{\pi\left(r_{2}^{4}-r_{1}^{4}\right)}{8|D|}\right]+O\left(\gamma a^{3 / 2}\right) \\
& =-\frac{1}{r_{1}}+\gamma\left[-\frac{r_{2}^{2} \log r_{2}-r_{1}^{2} \log r_{1}}{2}+\frac{a|D|}{4 \pi}+a|D| R(\xi, \xi)+\frac{\pi\left(r_{2}^{4}-r_{1}^{4}\right)}{8|D|}\right]+O(1) .
\end{aligned}
$$

To reach the second last line we have used the fact that $r_{1}=O\left(a^{1 / 2}\right)$. At each $\xi+r_{2} e^{i \theta}$ on the outer circle

$$
\begin{aligned}
\frac{1}{r_{2}} & +\gamma v\left(\xi+r_{2} e^{i \theta}\right) \\
& =\frac{1}{r_{2}}+\gamma\left[-\frac{r_{2}^{2}-r_{1}^{2}}{2} \log r_{2}+\pi\left(r_{2}^{2}-r_{1}^{2}\right) R\left(\xi+r_{2} e^{i \theta}, \xi\right)+\frac{\pi\left(r_{2}^{4}-r_{1}^{4}\right)}{8|D|}\right] \\
& =\frac{1}{r_{2}}+\gamma\left[-\frac{a|D|}{2 \pi} \log r_{2}+a|D| R\left(\xi+r_{2} e^{i \theta}, \xi\right)+\frac{\pi\left(r_{2}^{4}-r_{1}^{4}\right)}{8|D|}\right] \\
& =\frac{1}{r_{2}}+\gamma\left[-\frac{a|D|}{2 \pi} \log r_{2}+a|D| R(\xi, \xi)+\frac{\pi\left(r_{2}^{4}-r_{1}^{4}\right)}{8|D|}\right]+O\left(\gamma a^{3 / 2}\right) \\
& =\frac{1}{r_{2}}+\gamma\left[-\frac{a|D|}{2 \pi} \log r_{2}+a|D| R(\xi, \xi)+\frac{\pi\left(r_{2}^{4}-r_{1}^{4}\right)}{8|D|}\right]+O(1) .
\end{aligned}
$$

This proves the lemma.

Lemma 3.2 The value of $J$ at $R$ is

$$
\begin{aligned}
J(R)= & 2 \pi\left(r_{1}+r_{2}\right) \\
& +\frac{\gamma}{2}\left[-\frac{\pi r_{1}^{4} \log r_{1}}{2}-\frac{\pi r_{2}^{4} \log r_{2}}{2}+\pi r_{1}^{2} r_{2}^{2} \log r_{2}\right. \\
& -\frac{a|D| r_{1}^{2}}{4}+\frac{a^{2}|D|^{2}}{8 \pi}+a^{2}|D|^{2} R(\xi, \xi) \\
& \left.+\frac{a^{2}|D|}{4}\left(r_{1}^{2}+r_{2}^{2}\right)\right] .
\end{aligned}
$$

Proof. Let $v=(-\Delta)^{-1}\left(\chi_{R}-a\right)=v_{2}-v_{1}$ as in the proof of Lemma 3.1. The local part of $J(R)$ is just the arc length

$$
2 \pi r_{1}+2 \pi r_{2} .
$$

The nonlocal part of $J(R)$ is

$$
\begin{aligned}
& \frac{\gamma}{2} \int_{D}\left|(-\Delta)^{-1 / 2}\left(\chi_{R}-a\right)\right|^{2} d x \\
& \quad=\frac{\gamma}{2} \int_{D}\left(\chi_{R}-a\right) v(x) d x=\frac{\gamma}{2} \int_{D} \chi_{R} v(x) d x=\frac{\gamma}{2} \int_{R} v(x) d x \\
& \quad=\frac{\gamma}{2} \int_{B_{2} \backslash B_{1}}\left(v_{2}(x)-v_{1}(x)\right) d x=\frac{\gamma}{2}\left[\int_{B_{1}} v_{1}+\int_{B_{2}} v_{2}-\int_{B_{1}} v_{2}-\int_{B_{2}} v_{1}\right] \\
& \quad=\frac{\gamma}{2}[I+I I+I I I+I V]
\end{aligned}
$$


From the definition of $P_{k}$ one finds that

$$
\int_{B_{k}} P_{k}(x) d x=-\frac{\pi r_{k}^{4} \log r_{k}}{2}+\frac{\pi r_{k}^{4}}{8} .
$$

For the integral of $Q_{k}$, note that, since $\Delta Q_{k}(\cdot, \xi)=\frac{\pi r_{k}^{2}}{|D|}, Q_{k}(x, \xi)-\frac{\pi r_{k}^{2}}{4|D|}|x-\xi|^{2}$ is harmonic in $x$. By the Mean Value Theorem for harmonic functions

$$
\begin{aligned}
\int_{B_{k}} Q_{k}(x, \xi) d x & =\int_{B_{k}}\left(Q_{k}(x, \xi)-\frac{\pi r_{k}^{2}}{4|D|}|x-\xi|^{2}\right) d x+\int_{B_{k}} \frac{\pi r_{k}^{2}}{4|D|}|x-\xi|^{2} d x \\
& =\pi r_{k}^{2} Q_{k}(\xi, \xi)+\frac{\pi^{2} r_{k}^{6}}{8|D|}=\pi^{2} r_{k}^{4} R(\xi, \xi)+\frac{\pi^{2} r_{k}^{6}}{4|D|}
\end{aligned}
$$

Therefore from (3.8) and (3.9)

$$
\begin{gathered}
I=-\frac{\pi r_{1}^{4} \log r_{1}}{2}+\frac{\pi r_{1}^{4}}{8}+\pi^{2} r_{1}^{4} R(\xi, \xi)+\frac{\pi^{2} r_{1}^{6}}{4|D|}, \\
I I=-\frac{\pi r_{2}^{4} \log r_{2}}{2}+\frac{\pi r_{2}^{4}}{8}+\pi^{2} r_{2}^{4} R(\xi, \xi)+\frac{\pi^{2} r_{2}^{6}}{4|D|} .
\end{gathered}
$$

Next note that

$$
\begin{aligned}
I I I & =-\int_{B_{1}} v_{2} d x=-\int_{D} \chi_{B_{1}} v_{2}=-\int_{D}\left(\chi_{B_{1}}-\frac{\pi r_{1}^{2}}{|D|}\right) v_{2} d x \\
& =\int_{D} \Delta v_{1} v_{2}=-\int_{D} \nabla v_{1} \cdot \nabla v_{2} d x=\int_{D} v_{1} \Delta v_{2} d x=-\int_{B_{2}} v_{1} d x=I V .
\end{aligned}
$$

Therefore,

$$
\begin{aligned}
I I I & =I V=-\int_{B_{1}} v_{2} d x=-\int_{B_{1}} P_{2} d x-\int_{B_{1}} Q_{2} d x \\
& =\frac{\pi r_{1}^{4}}{8}-\pi r_{1}^{2}\left(\frac{r_{2}^{2}}{4}-\frac{r_{2}^{2}}{2} \log r_{2}\right)-\int_{B_{1}}\left(Q_{2}(x)-\frac{\pi r_{2}^{2}}{4|D|}|x-\xi|^{2}\right) d x-\int_{B_{1}} \frac{\pi r_{2}^{2}}{4|D|}|x-\xi|^{2} d x \\
& =\frac{\pi r_{1}^{4}}{8}-\pi r_{1}^{2}\left(\frac{r_{2}^{2}}{4}-\frac{r_{2}^{2}}{2} \log r_{2}\right)-\pi r_{1}^{2} Q_{2}(\xi)-\frac{\pi^{2} r_{2}^{2} r_{1}^{4}}{8|D|} \\
& =\frac{\pi r_{1}^{4}}{8}-\pi r_{1}^{2}\left(\frac{r_{2}^{2}}{4}-\frac{r_{2}^{2}}{2} \log r_{2}\right)-\pi^{2} r_{1}^{2} r_{2}^{2} R(\xi, \xi)-\frac{\pi^{2} r_{2}^{2} r_{1}^{4}}{8|D|}-\frac{\pi^{2} r_{1}^{2} r_{2}^{4}}{8|D|}
\end{aligned}
$$

Finally we sum all these identities and use the fact $\pi r_{2}^{2}-\pi r_{1}^{2}=a|D|$, to deduce the conclusion.

\section{Perturbed rings}

A perturbed ring $E_{\phi}$ is characterized by a pair of $2 \pi$ periodic functions $\phi(\theta)=\left(\phi_{1}(\theta), \phi_{2}(\theta)\right)$ so that

$$
E_{\phi}=\left\{\xi+\alpha e^{i \theta}: \theta \in[0,2 \pi], \alpha \in\left(\sqrt{r_{1}^{2}+\phi_{1}(\theta)}, \sqrt{r_{2}^{2}+\phi_{2}(\theta)}\right)\right\}
$$

and the boundaries of the perturbed ring $E_{\phi}$ are two curves parametrized by $\theta: \xi+\sqrt{r_{1}^{2}+\phi_{1}(\theta)} e^{i \theta}$, which is the perturbed inner circle, and $\xi+\sqrt{r_{2}^{2}+\phi_{2}(\theta)} e^{i \theta}$, the perturbed outer circle. We will 
restrict the size of $\phi_{1}, \phi_{2}$ so that $r_{1}^{2}+\phi_{1}, r_{2}^{2}+\phi_{2}$ are always positive. Moreover it is always assumed that $\phi \perp(-1,1)$, i.e.

$$
-\int_{0}^{2 \pi} \phi_{1}(\theta) d \theta+\int_{0}^{2 \pi} \phi_{2}(\theta) d \theta=0 .
$$

This ensures that the size of $E_{\phi}$ remains $a|D|$ :

$$
\left|E_{\phi}\right|=\int_{0}^{2 \pi} \int_{\sqrt{r_{1}^{2}+\phi_{1}(\theta)}}^{\sqrt{r_{2}^{2}+\phi_{2}(\theta)}} r d r d \theta=\int_{0}^{2 \pi} \frac{r_{2}^{2}+\phi_{2}(\theta)-r_{1}^{2}-\phi_{1}(\theta)}{2} d \theta=\pi r_{2}^{2}-\pi r_{1}^{2}=a|D| .
$$

The arc-length of $\partial_{D} E_{\phi}$ can be expressed as

$$
\left|D \chi_{E_{\phi}}\right|(D)=\int_{0}^{2 \pi} \sqrt{r_{1}^{2}+\phi_{1}(\theta)+\frac{\left(\phi_{1}^{\prime}(\theta)\right)^{2}}{4\left(r_{1}^{2}+\phi_{1}(\theta)\right)}} d \theta+\int_{0}^{2 \pi} \sqrt{r_{2}^{2}+\phi_{2}(\theta)+\frac{\left(\phi_{2}^{\prime}(\theta)\right)^{2}}{4\left(r_{2}^{2}+\phi_{2}(\theta)\right)}} d \theta .
$$

Calculating the variations of (4.3) we obtain two quasi-linear operators

$$
\mathcal{H}_{k}\left(\phi_{k}\right)(\theta)=\frac{r_{k}^{2}+\phi_{k}(\theta)+\frac{3\left(\phi_{k}^{\prime}(\theta)\right)^{2}}{4\left(r_{k}^{2}+\phi_{k}(\theta)\right)}-\frac{\phi_{k}^{\prime \prime}(\theta)}{2}}{2\left(r_{k}^{2}+\phi_{k}(\theta)+\frac{\left(\phi_{k}^{\prime}(\theta)\right)^{2}}{4\left(r_{k}^{2}+\phi_{k}(\theta)\right)}\right)^{3 / 2}}, k=1,2 .
$$

Note that $\mathcal{H}_{2}$ gives half of the curvature of the perturbed outer boundary viewed from $E_{\phi}$. However $\mathcal{H}_{1}$ is negative half of the curvature of the perturbed inner boundary viewed from $E_{\phi}$.

The nonlocal part of $J$ in (1.2) may be written in terms of $\phi$ as

$$
\begin{aligned}
& \frac{\gamma}{2} \int_{D}\left|(-\Delta)^{-1 / 2}\left(\chi_{E_{\phi}}-a\right)\right|^{2} d x=\frac{\gamma}{2} \int_{E_{\phi}} \int_{E_{\phi}} G(x, y) d x d y \\
& \quad=\frac{\gamma}{2} \int_{0}^{2 \pi} d \theta \int_{\sqrt{r_{1}^{2}+\phi_{1}(\theta)}}^{\sqrt{r_{2}^{2}+\phi_{2}(\theta)}} d r \int_{0}^{2 \pi} d \omega \int_{\sqrt{r_{1}^{2}+\phi_{1}(\omega)}}^{\sqrt{r_{2}^{2}+\phi_{2}(\omega)}} d t G\left(\xi+r e^{i \theta}, \xi+t e^{i \omega}\right) r t .
\end{aligned}
$$

The variation of (4.5) with respect to $\phi_{1}$ is

$$
-\frac{\gamma}{2}(-\Delta)^{-1}\left(\chi_{E_{\phi}}-a\right)\left(\xi+\sqrt{r_{1}^{2}+\phi_{1}(\theta)} e^{i \theta}\right)=-\frac{\gamma}{2} \int_{E_{\phi}} G\left(\xi+\sqrt{r_{1}^{2}+\phi_{1}(\theta)} e^{i \theta}, y\right) d y ;
$$

and the variation of (4.5) with respect to $\phi_{2}$ is

$$
\frac{\gamma}{2}(-\Delta)^{-1}\left(\chi_{E_{\phi}}-a\right)\left(\xi+\sqrt{r_{2}^{2}+\phi_{2}(\theta)} e^{i \theta}\right)=\frac{\gamma}{2} \int_{E_{\phi}} G\left(\xi+\sqrt{r_{2}^{2}+\phi_{2}(\theta)} e^{i \theta}, y\right) d y .
$$

Under the constraint (4.2) the Euler-Lagrange equations of $J$ are

$$
\begin{aligned}
& \mathcal{H}_{1}\left(\phi_{1}\right)(\theta)-\frac{\gamma}{2}(-\Delta)^{-1}\left(\chi_{E_{\phi}}-a\right)\left(\xi+\sqrt{r_{1}^{2}+\phi_{1}(\theta)} e^{i \theta}\right)=\lambda \\
& \mathcal{H}_{2}\left(\phi_{2}\right)(\theta)+\frac{\gamma}{2}(-\Delta)^{-1}\left(\chi_{E_{\phi}}-a\right)\left(\xi+\sqrt{r_{2}^{2}+\phi_{2}(\theta)} e^{i \theta}\right)=-\lambda
\end{aligned}
$$

in terms of $\phi_{1}$ and $\phi_{2}$.

Remark 4.1 Note that (4.9) differs from (1.1) by a half while (4.8) differs from (1.1) by a negative half. 
Let us define

$$
\begin{aligned}
& \mathcal{A}_{1}(\phi)(\theta)=\frac{\gamma}{4 \pi} \int_{0}^{2 \pi} \int_{\sqrt{r_{1}^{2}+\phi_{1}(\omega)}}^{\sqrt{r_{2}^{2}+\phi_{2}(\omega)}} \log \left|\sqrt{r_{1}^{2}+\phi_{1}(\theta)} e^{i \theta}-t e^{i \omega}\right| t d t d \omega \\
& \mathcal{B}_{1}(\phi)(\theta)=-\frac{\gamma}{2} \int_{0}^{2 \pi} \int_{\sqrt{r_{1}^{2}+\phi_{1}(\omega)}}^{\sqrt{r_{2}^{2}+\phi_{2}(\omega)}} R\left(\xi+\sqrt{r_{1}^{2}+\phi_{1}(\theta)} e^{i \theta}, \xi+t e^{i \omega}\right) t d t d \omega \\
& \mathcal{A}_{2}(\phi)(\theta)=-\frac{\gamma}{4 \pi} \int_{0}^{2 \pi} \int_{\sqrt{r_{1}^{2}+\phi_{1}(\omega)}}^{\sqrt{r_{2}^{2}+\phi_{2}(\omega)}} \log \left|\sqrt{r_{2}^{2}+\phi_{2}(\theta)} e^{i \theta}-t e^{i \omega}\right| t d t d \omega \\
& \mathcal{B}_{2}(\phi)(\theta)=\frac{\gamma}{2} \int_{0}^{2 \pi} \int_{\sqrt{r_{1}^{2}+\phi_{1}(\omega)}}^{\sqrt{r_{2}^{2}+\phi_{2}(\omega)}} R\left(\xi+\sqrt{r_{2}^{2}+\phi_{2}(\theta)} e^{i \theta}, \xi+t e^{i \omega}\right) t d t d \omega,
\end{aligned}
$$

so that (4.8) and (4.9) become

$$
\mathcal{H}_{1}\left(\phi_{1}\right)+\mathcal{A}_{1}(\phi)+\mathcal{B}_{1}(\phi)=\lambda ; \mathcal{H}_{2}\left(\phi_{2}\right)+\mathcal{A}_{2}(\phi)+\mathcal{B}_{2}(\phi)=-\lambda .
$$

Note that the operators $\mathcal{H}_{k}$ and $\mathcal{A}_{k}$ are independent of $\xi$ while the operators $\mathcal{B}_{k}$ do depend on $\xi$.

Let $\mathcal{S}=\left(\mathcal{S}_{1}, \mathcal{S}_{2}\right)$ be the operator that appears on the left side of (4.14) projected to $\{(-1,1)\}^{\perp}$, i.e.

$$
\mathcal{S}_{k}(\phi)=\mathcal{H}_{k}\left(\phi_{k}\right)+\mathcal{A}_{k}(\phi)+\mathcal{B}_{k}(\phi)+(-1)^{k} \lambda(\phi)
$$

for $k=1,2$. Here $\lambda(\phi)$ is a number so chosen that $\mathcal{S}(\phi) \perp(-1,1)$, i.e.

$$
\int_{0}^{2 \pi}\left(-\mathcal{S}_{1}(\phi)+\mathcal{S}_{2}(\phi)\right) d \theta=0 .
$$

Now $E_{\phi}$ is a solution of (1.1) (and of course (4.14)) if and only if

$$
\mathcal{S}(\phi)=0 .
$$

The operator $\mathcal{S}=\left(\mathcal{S}_{1}, \mathcal{S}_{2}\right)$ maps from

$$
\mathcal{X}=\left\{\phi=\left[\begin{array}{l}
\phi_{1}(\theta) \\
\phi_{2}(\theta)
\end{array}\right]: \phi_{k} \in H^{2}\left(S^{1}\right), k=1,2, \phi \perp\left[\begin{array}{r}
-1 \\
1
\end{array}\right]\right\}
$$

to

$$
\mathcal{Y}=\left\{q=\left[\begin{array}{l}
q_{1}(\theta) \\
q_{2}(\theta)
\end{array}\right]: q_{k} \in L^{2}\left(S^{1}\right), k=1,2, q \perp\left[\begin{array}{r}
-1 \\
1
\end{array}\right]\right\}
$$

The first Fréchet derivative of $\mathcal{S}$ is given by

$$
\begin{aligned}
\mathcal{H}_{k}^{\prime}\left(\phi_{k}\right)\left(u_{k}\right)= & \mathcal{H}_{k, \phi_{k}}(\phi) u_{k}+\mathcal{H}_{k, \phi_{k}^{\prime}}(\phi) u_{k}^{\prime}+\mathcal{H}_{k, \phi_{k}^{\prime \prime}}(\phi) u_{k}^{\prime \prime} \\
\mathcal{A}_{1}^{\prime}(\phi)(u)(\theta)= & -\frac{\gamma}{8 \pi} \int_{0}^{2 \pi} u_{1}(\omega) \log \left|\sqrt{r_{1}^{2}+\phi_{1}(\theta)} e^{i \theta}-\sqrt{r_{1}^{2}+\phi_{1}(\omega)} e^{i \omega}\right| d \omega \\
& +\frac{\gamma u_{1}(\theta)}{8 \pi \sqrt{r_{1}^{2}+\phi_{1}(\theta)}} \int_{\tilde{E}_{\phi}} \frac{\left(\sqrt{r_{1}^{2}+\phi_{1}(\theta)} e^{i \theta}-y\right) \cdot e^{i \theta}}{\left|\sqrt{r_{1}^{2}+\phi_{1}(\theta)} e^{i \theta}-y\right|^{2}} d y \\
& +\frac{\gamma}{8 \pi} \int_{0}^{2 \pi} u_{2}(\omega) \log \left|\sqrt{r_{1}^{2}+\phi_{1}(\theta)} e^{i \theta}-\sqrt{r_{2}^{2}+\phi_{2}(\omega)} e^{i \omega}\right| d \omega . \\
\mathcal{B}_{1}^{\prime}(\phi)(u)(\theta)= & \frac{\gamma}{4} \int_{0}^{2 \pi} u_{1}(\omega) R\left(\xi+\sqrt{r_{1}^{2}+\phi_{1}(\theta)} e^{i \theta}, \xi+\sqrt{r_{1}^{2}+\phi_{1}(\omega)} e^{i \omega}\right) d \omega
\end{aligned}
$$




$$
\begin{aligned}
& -\frac{\gamma u_{1}(\theta)}{4 \sqrt{r_{1}^{2}+\phi_{1}(\theta)}} \int_{E_{\phi}} \nabla R\left(\xi+\sqrt{r_{1}^{2}+\phi_{1}(\theta)} e^{i \theta}, y\right) \cdot e^{i \theta} d y . \\
& -\frac{\gamma}{4} \int_{0}^{2 \pi} u_{2}(\omega) R\left(\xi+\sqrt{r_{1}^{2}+\phi_{1}(\theta)} e^{i \theta}, \xi+\sqrt{r_{2}^{2}+\phi_{2}(\omega)} e^{i \omega}\right) d \omega \\
\mathcal{A}_{2}^{\prime}(\phi)(u)(\theta)= & \frac{\gamma}{8 \pi} \int_{0}^{2 \pi} u_{1}(\omega) \log \left|\sqrt{r_{2}^{2}+\phi_{2}(\theta)} e^{i \theta}-\sqrt{r_{1}^{2}+\phi_{1}(\omega)} e^{i \omega}\right| d \omega \\
& -\frac{\gamma}{8 \pi} \int_{0}^{2 \pi} u_{2}(\omega) \log \left|\sqrt{r_{2}^{2}+\phi_{2}(\theta)} e^{i \theta}-\sqrt{r_{2}^{2}+\phi_{2}(\omega)} e^{i \omega}\right| d \omega \\
& -\frac{\gamma u_{2}(\theta)}{8 \pi \sqrt{r_{2}^{2}+\phi_{2}(\theta)}} \int_{\tilde{E}_{\phi}} \frac{\left(\sqrt{r_{2}^{2}+\phi_{2}(\theta)} e^{i \theta}-y\right) \cdot e^{i \theta}}{\left|\sqrt{r_{2}^{2}+\phi_{2}(\theta)} e^{i \theta}-y\right|^{2}} d y \\
\mathcal{B}_{2}^{\prime}(\phi)(u)(\theta)= & -\frac{\gamma}{4} \int_{0}^{2 \pi} u_{1}(\omega) R\left(\xi+\sqrt{r_{2}^{2}+\phi_{2}(\theta)} e^{i \theta}, \xi+\sqrt{r_{1}^{2}+\phi_{1}(\omega)} e^{i \omega}\right) d \omega \\
& +\frac{\gamma}{4} \int_{0}^{2 \pi} u_{2}(\omega) R\left(\xi+\sqrt{r_{2}^{2}+\phi_{2}(\theta)} e^{i \theta}, \xi+\sqrt{r_{2}^{2}+\phi_{2}(\omega)} e^{i \omega}\right) d \omega \\
& +\frac{\gamma u_{2}(\theta)}{4 \sqrt{r_{2}^{2}+\phi_{2}(\theta)}} \int_{E_{\phi}} \nabla R\left(\xi+\sqrt{r_{2}^{2}+\phi_{2}(\theta)} e^{i \theta}, y\right) \cdot e^{i \theta} d y .
\end{aligned}
$$

Here $\tilde{E}_{\phi}$ in $\mathcal{A}_{k}^{\prime}, k=1,2$, is a shift of $E_{\phi}$ so that $\tilde{E}_{\phi}$ is centered at 0 , i.e. $\tilde{E}_{\phi}=E_{\phi}-\xi$. The derivative of the operator $\lambda$ is so chosen that

$$
\mathcal{S}_{k}^{\prime}(\phi)=\mathcal{H}_{k}^{\prime}(\phi)+\mathcal{A}_{k}^{\prime}(\phi)+\mathcal{B}_{k}^{\prime}(\phi)+(-1)^{k} \lambda^{\prime}(\phi), \mathcal{S}^{\prime}(\phi)(u) \perp(-1,1) .
$$

We have abused the notations a bit in (4.20). The operator $\mathcal{H}_{k}$ is also viewed as a function of $\phi$, $\phi^{\prime}$ and $\phi^{\prime \prime}$. The derivatives of $\mathcal{H}_{k}$ with respect to $\phi_{k}, \phi_{k}^{\prime}$ and $\phi_{k}^{\prime \prime}$ are denoted by $\mathcal{H}_{k, \phi_{k}}, \mathcal{H}_{k, \phi_{k}^{\prime}}$ and $\mathcal{H}_{k, \phi_{k}^{\prime \prime}}$ respectively. In $\mathcal{B}_{k}^{\prime}, \nabla R$ is the gradient of $R$ with respect to its first argument.

\section{Linear analysis}

Let $\mathcal{L}$ be the linearized operator of $\mathcal{S}$ at $\phi=0$, i.e, at $E=R=B_{2} \backslash B_{1}$ :

$$
\mathcal{L}=\mathcal{S}^{\prime}(0)
$$

Going back to (4.20), (4.22),(4.23), (4.24) and (4.24) we find that

$$
\begin{aligned}
\mathcal{H}_{k}^{\prime}(0)\left(u_{k}\right)= & -\frac{1}{4 r_{k}^{3}}\left(u_{k}^{\prime \prime}+u_{k}\right), \\
\mathcal{A}_{1}^{\prime}(0)(u)(\theta)= & -\frac{\gamma}{8 \pi} \int_{0}^{2 \pi} u_{1}(\omega) \log \left|r_{1} e^{i \theta}-r_{1} e^{i \omega}\right| d \omega+\frac{\gamma}{8 \pi} \int_{0}^{2 \pi} u_{2}(\omega) \log \left|r_{1} e^{i \theta}-r_{2} e^{i \omega}\right| d \omega, \\
\mathcal{B}_{1}^{\prime}(0)(u)(\theta)= & \frac{\gamma}{4} \int_{0}^{2 \pi} u_{1}(\omega) R\left(\xi+r_{1} e^{i \theta}, \xi+r_{1} e^{i \omega}\right) d \omega-\frac{\gamma u_{1}(\theta)}{4 r_{1}} \int_{R} \nabla R\left(\xi+r_{1} e^{i \theta}, y\right) \cdot e^{i \theta} d y \\
& -\frac{\gamma}{4} \int_{0}^{2 \pi} u_{2}(\omega) R\left(\xi+r_{1} e^{i \theta}, \xi+r_{2} e^{i \omega}\right) d \omega \\
\mathcal{A}_{2}^{\prime}(0)(u)(\theta)= & \frac{\gamma}{8 \pi} \int_{0}^{2 \pi} u_{1}(\omega) \log \left|r_{2} e^{i \theta}-r_{1} e^{i \omega}\right| d \omega-\frac{\gamma}{8 \pi} \int_{0}^{2 \pi} u_{2}(\omega) \log \left|r_{2} e^{i \theta}-r_{2} e^{i \omega}\right| d \omega \\
& -\frac{\gamma u_{2}(\theta)}{8}\left(1-\frac{r_{1}^{2}}{r_{2}^{2}}\right),
\end{aligned}
$$




$$
\begin{aligned}
\mathcal{B}_{2}^{\prime}(0)(u)(\theta)= & -\frac{\gamma}{4} \int_{0}^{2 \pi} u_{1}(\omega) R\left(\xi+r_{2} e^{i \theta}, \xi+r_{1} e^{i \omega}\right) d \omega+\frac{\gamma}{4} \int_{0}^{2 \pi} u_{2}(\omega) R\left(\xi+r_{2} e^{i \theta}, \xi+r_{2} e^{i \omega}\right) d \omega \\
& +\frac{\gamma u_{2}(\theta)}{4 r_{2}} \int_{R} \nabla R\left(\xi+r_{2} e^{i \theta}, y\right) \cdot e^{i \theta} d y .
\end{aligned}
$$

The derivation of $\mathcal{A}_{k}^{\prime}(0)$ is explained in more detail in Appendix A.

Let us separate $\mathcal{L}$ to a dominant part $\mathcal{L}_{1}$ and a minor part $\mathcal{L}_{2}$. We define $\mathcal{L}_{1, k}$, the $k$-th component of $\mathcal{L}_{1}$, to be

$$
\begin{aligned}
\mathcal{L}_{1,1}(u)(\theta)= & -\frac{1}{4 r_{1}^{3}}\left(u_{1}^{\prime \prime}(\theta)+u_{1}(\theta)\right) \\
& -\frac{\gamma}{8 \pi} \int_{0}^{2 \pi} u_{1}(\omega) \log \left|r_{1} e^{i \theta}-r_{1} e^{i \omega}\right| d \omega \\
& +\frac{\gamma}{8 \pi} \int_{0}^{2 \pi} u_{2}(\omega) \log \left|r_{1} e^{i \theta}-r_{2} e^{i \omega}\right| d \omega \\
& -l_{1}(u) \\
\mathcal{L}_{1,2}(u)(\theta)= & -\frac{1}{4 r_{2}^{3}}\left(u_{2}^{\prime \prime}(\theta)+u_{2}(\theta)\right) \\
& +\frac{\gamma}{8 \pi} \int_{0}^{2 \pi} u_{1}(\omega) \log \left|r_{2} e^{i \theta}-r_{1} e^{i \omega}\right| d \omega \\
& -\frac{\gamma}{8 \pi} \int_{0}^{2 \pi} u_{2}(\omega) \log \left|r_{2} e^{i \theta}-r_{2} e^{i \omega}\right| d \omega \\
& -\frac{\gamma u_{2}(\theta)}{8}\left(1-\frac{r_{1}^{2}}{r_{2}^{2}}\right) \\
& +l_{1}(u) .
\end{aligned}
$$

The real valued linear operator $l_{1}$ is independent of $k$. It is so chosen that $\mathcal{L}_{1}$ maps from $\mathcal{X}$ to $\mathcal{Y}$. The rest of $\mathcal{L}$ is denoted by $\mathcal{L}_{2}$.

From now on we set

$$
r=\frac{r_{1}}{r_{2}}<1 .
$$

We are more interested in the operator $\Pi \mathcal{L}$ and $\Pi \mathcal{L}_{1}$ where $\Pi$ is the orthogonal projection operator from $\mathcal{Y}$ to

$$
\mathcal{Y}_{*}=\left\{q=\left[\begin{array}{l}
q_{1} \\
q_{2}
\end{array}\right] \in \mathcal{Y}: q \perp \cos \theta\left[\begin{array}{l}
r \\
1
\end{array}\right], q \perp \sin \theta\left[\begin{array}{l}
r \\
1
\end{array}\right], q \perp\left[\begin{array}{l}
1 \\
1
\end{array}\right]\right\} .
$$

The operator $\Pi \mathcal{L}$ is defined on

$$
\mathcal{X}_{*}=\left\{u=\left[\begin{array}{l}
u_{1} \\
u_{2}
\end{array}\right] \in \mathcal{X}: u \perp \cos \theta\left[\begin{array}{l}
r \\
1
\end{array}\right], u \perp \sin \theta\left[\begin{array}{l}
r \\
1
\end{array}\right], u \perp\left[\begin{array}{l}
1 \\
1
\end{array}\right]\right\} \subset \mathcal{Y}_{*} .
$$

Since every element of $\mathcal{X}$ (and $\mathcal{Y}$ ) is perpendicular to $(-1,1)$, if $u=\left(u_{1}, u_{2}\right) \in \mathcal{X}_{*}\left(\right.$ or $\left.\mathcal{Y}_{*}\right)$, it must be perpendicular to any constant vector, i.e.

$$
\int_{0}^{2 \pi} u_{k}(\theta) d \theta=0, \quad k=1,2 .
$$

Consequently

$$
l_{1}(u)=0, \text { if } u \in \mathcal{X}_{*} .
$$


Lemma 5.1 1. $\|u\|_{H^{2}} \leq C a^{3 / 2}\left\|\mathcal{L}_{1}(u)\right\|_{L^{2}}$ for all $u \in \mathcal{X}_{*}$.

2. Under the condition of Theorem 2.2 that $\left(S_{0,1}, \Gamma\right)$ lies below all the $W_{n}$ 's, we have $\|u\|_{H^{1}}^{2} \leq$ $C a^{3 / 2}\left\langle\mathcal{L}_{1}(u), u\right\rangle$ for all $u \in \mathcal{X}_{*}$.

Proof. The spectrum of $\Pi \mathcal{L}_{1}$ can be computed explicitly using Fourier series. The Fourier space of $\mathcal{X}_{*}$ is

$$
\begin{aligned}
\widehat{\mathcal{X}_{*}}= & \left\{\left(\left\{l_{n, 1}\right\},\left\{l_{n, 2}\right\}\right): \sum_{n=-\infty}^{\infty}\left|l_{n, k}\right|^{2}<\infty, k=1,2, \quad\left(l_{0,1}, l_{0,2}\right)=(0,0),\right. \\
& \left.\left(l_{1,1}, l_{1,2}\right) \perp(r, 1),\left(l_{-1,1}, l_{-1,2}\right) \perp(r, 1)\right\}
\end{aligned}
$$

Let

$$
\widehat{u_{k}}(n)=\int_{0}^{2 \pi} u_{k}(\theta) e^{-i n \theta} d \theta
$$

be the $n$-th Fourier coefficient of $u_{k}$, then when $n \neq 0$

$$
\begin{aligned}
& \widehat{\mathcal{L}_{1,1}(u)}(n)=\left(\frac{n^{2}-1}{4 r_{1}^{3}}+\frac{\gamma}{8|n|}\right) \widehat{u_{1}}(n)-\frac{\gamma r^{|n|}}{8|n|} \widehat{u_{2}}(n) \\
& \widehat{\mathcal{L}_{1,2}(u)}(n)=-\frac{\gamma r^{|n|}}{8|n|} \widehat{u_{1}}(n)+\left[\frac{n^{2}-1}{4 r_{2}^{3}}+\frac{\gamma}{8}\left(\frac{1}{|n|}-1+r^{2}\right)\right] \widehat{u_{2}}(n) .
\end{aligned}
$$

To derive (5.9) and (5.10) we have used the well known formula

$$
\log \left|1-e^{i \theta}\right|=-\sum_{n=1}^{\infty} \frac{\cos n \theta}{n} .
$$

See for instance Tolstov [27, Page 93]. We have also used the formula

$$
\log \left|1-r e^{i \theta}\right|=-\sum_{n=1}^{\infty} \frac{r^{n} \cos n \theta}{n}, r \in(0,1) .
$$

A short derivation of (5.12) is given in Appendix B.

Note that $\Pi \mathcal{L}_{1}=\mathcal{L}_{1}$ on $\mathcal{X}_{*}$, which follows from the facts

$$
\left[\begin{array}{c}
\widehat{\mathcal{L}_{1,1}(u)}(1) \\
\mathcal{L}_{1,2}(u)(1)
\end{array}\right] \perp\left[\begin{array}{l}
r \\
1
\end{array}\right],\left[\begin{array}{c}
\widehat{\mathcal{L}_{1,1}(u)}(-1) \\
\widehat{\mathcal{L}_{1,2}(u)(-1)}
\end{array}\right] \perp\left[\begin{array}{l}
r \\
1
\end{array}\right] .
$$

We define, for $n \geq 1$,

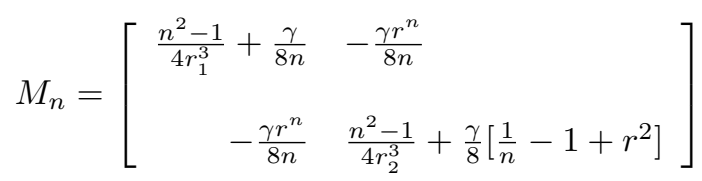

For $n=1$,

$$
M_{1}=\left[\begin{array}{rr}
\frac{\gamma}{8} & -\frac{\gamma r}{8} \\
-\frac{\gamma r}{8} & \frac{\gamma r^{2}}{8}
\end{array}\right]
$$

has two eigenvalues. One is $\lambda_{1,1}=0$, with eigenvectors

$$
\cos \theta\left[\begin{array}{l}
r \\
1
\end{array}\right], \quad \sin \theta\left[\begin{array}{l}
r \\
1
\end{array}\right] .
$$


However they are perpendicular to $\mathcal{X}_{*}$ and therefore discarded.

The second eigenvalue is $\lambda_{1,2}=\frac{\gamma\left(1+r^{2}\right)}{8}>C a^{-3 / 2}$ for some $C>0$ independent of $a$, with eigenvectors

$$
\cos \theta\left[\begin{array}{r}
1 \\
-r
\end{array}\right], \quad \sin \theta\left[\begin{array}{r}
1 \\
-r
\end{array}\right]
$$

which are in $\mathcal{X}_{*}$.

For $n \geq 2$, denote the $(1,1)$ entry of $M_{n}$ by $c_{1},(2,2)$ entry by $c_{2}$, and $(1,2)$ and $(2,1)$ entries by d. Then

$$
\operatorname{det}\left(\lambda I-M_{n}\right)=\lambda^{2}-\left(c_{1}+c_{2}\right) \lambda+c_{1} c_{2}-d^{2} .
$$

Let $\lambda_{n, 1}, \lambda_{n, 2}$ be the two eigenvalues of $M_{n}$, then we find that

$$
\begin{aligned}
\lambda_{n, 1} & =\frac{c_{1}+c_{2}+\sqrt{\left(c_{1}-c_{2}\right)^{2}+4 d^{2}}}{2}, \\
\lambda_{n, 2} & =\frac{c_{1}+c_{2}-\sqrt{\left(c_{1}-c_{2}\right)^{2}+4 d^{2}}}{2} .
\end{aligned}
$$

It is obvious that $c_{1}>c_{2}$, therefore

$$
\lambda_{n, 1}>\frac{c_{1}+c_{2}+c_{1}-c_{2}}{2}=c_{1}=\frac{n^{2}-1}{4 r_{1}^{3}}+\frac{\gamma}{8 n}>C n^{2} a^{-3 / 2}>0
$$

where $C>0$ is independent of $a$.

It remains to study $\lambda_{n, 2}$. Let us introduce scaled variables $R_{j}$ and $\Gamma$ where

$$
R_{j}=\left(\frac{a|D|}{\pi}\right)^{-1 / 2} r_{j}, j=1,2 ; \quad \Gamma=\left(\frac{a|D|}{\pi}\right)^{3 / 2} \gamma .
$$

The constraint on $r_{j}$ now becomes

$$
R_{2}^{2}-R_{1}^{2}=1
$$

The range (3.3) for $r_{1}$ and $r_{2}$ implies that

$$
S_{0,1}-\delta_{2}<R_{1}<S_{0,1}+\delta_{2}
$$

The matrices $M_{n}$ can be written as

$$
M_{n}=\left(\frac{a|D|}{\pi}\right)^{-3 / 2}\left[\begin{array}{ll}
\frac{n^{2}-1}{4 R_{1}^{3}}+\frac{\Gamma}{8 n} & -\frac{\Gamma}{8 n}\left(\frac{R_{1}}{R_{2}}\right)^{n} \\
-\frac{\Gamma}{8 n}\left(\frac{R_{1}}{R_{2}}\right)^{n} & \frac{n^{2}-1}{4 R_{2}^{3}}+\frac{\Gamma}{8}\left[\frac{1}{n}-1+\left(\frac{R_{1}}{R_{2}}\right)^{2}\right]
\end{array}\right], \quad n \geq 2 .
$$

It is easy to see that asymptotically for fixed $R_{1}$ and $\Gamma$

$$
\lim _{n \rightarrow \infty} \frac{\lambda_{n, 1}}{\left(\frac{a|D|}{\pi}\right)^{-3 / 2}\left(\frac{n^{2}-1}{4 R_{1}^{3}}\right)}=1, \quad \lim _{n \rightarrow \infty} \frac{\lambda_{n, 2}}{\left(\frac{a|D|}{\pi}\right)^{-3 / 2}\left(\frac{n^{2}-1}{4 R_{2}^{3}}\right)}=1 .
$$

Note that the second eigenvalue $\lambda_{n, 2}$ is not zero if $\operatorname{det} M_{n} \neq 0$, and it is positive if $\operatorname{det} M_{n}>0$. The equation $\operatorname{det} M_{n}=0$ is quadratic in $\Gamma$ :

$$
\frac{1-r^{2 n}-n\left(1-r^{2}\right)}{64 n^{2}} \Gamma^{2}+\left[\frac{n^{2}-1}{32 n R_{2}^{3}}+\frac{n^{2}-1}{32 R_{1}^{3}}\left(\frac{1}{n}-1+r^{2}\right)\right] \Gamma+\frac{\left(n^{2}-1\right)^{2}}{16 R_{1}^{3} R_{2}^{3}}=0 .
$$

The graph of the left side, as a function of $\Gamma$, is a downward parabola. Its intersection with the vertical axis is $\left(0, \frac{\left(n^{2}-1\right)^{2}}{4 R_{1}^{3} R_{2}^{3}}\right)$. Therefore one root for $\Gamma$ is negative, and the other root is positive. 
We focus on the positive root which in Section 2 is denoted by $\tilde{\Gamma}_{n}\left(R_{1}\right)$. The first condition in Theorem 2.1 on $S_{0,1}$ and $\Gamma$ ensures that if $\delta_{2}$ is small, $\operatorname{det} M_{n} \neq 0$ and hence the second eigenvalue $\lambda_{n, 2}$ is not 0 . With the help of the asymptotic formulae (5.19) we find $C>0$, independent of $a$, such that

This implies that

$$
\lambda_{1,2}>C a^{-3 / 2}, \quad \frac{\left|\lambda_{n, k}\right|}{n^{2}}>C a^{-3 / 2}, k=1,2, n=2,3, \ldots
$$

$$
\|u\|_{H^{2}} \leq C a^{3 / 2}\left\|\mathcal{L}_{1}(u)\right\|_{L^{2}}
$$

for all $u \in \mathcal{X}_{*}$.

If we further assume that $\left(S_{0,1}, \Gamma\right)$ lies below all the $W_{n}$ 's, then $\left(R_{1}, \Gamma\right)$ also lies below all the $W_{n}$ 's, if we let $\delta_{2}$ be small enough. In this case $\operatorname{det} M_{n}>0$ and there exists $C>0$ such that

$$
\lambda_{1,2}>C a^{-3 / 2}, \quad \frac{\lambda_{n, k}}{n^{2}}>C a^{-3 / 2}, k=1,2, n=2,3, \ldots
$$

This implies that

$$
\|u\|_{H^{1}}^{2} \leq C a^{3 / 2}\left\langle\mathcal{L}_{1}(u), u\right\rangle
$$

This proves the lemma.

The second part $\mathcal{L}_{2}$ in $\mathcal{L}$ is a minor part.

Lemma 5.2 There exists $C>0$ independent of $\xi, r_{1}, r_{2}$ such that $\left\|\mathcal{L}_{2}(u)\right\|_{L^{2}} \leq C a^{-1}\|u\|_{L^{2}}$ for all $u \in \mathcal{X}_{*}$.

Proof. Recall $\mathcal{L}_{2}$ :

$$
\begin{aligned}
\mathcal{L}_{2,1}(u)(\theta)= & \frac{\gamma}{4} \int_{0}^{2 \pi} u_{1}(\omega) R\left(\xi+r_{1} e^{i \theta}, \xi+r_{1} e^{i \omega}\right) d \omega \\
& -\frac{\gamma u_{1}(\theta)}{4 r_{1}} \int_{R} \nabla R\left(\xi+r_{1} e^{i \theta}, y\right) \cdot e^{i \theta} d y \\
& -\frac{\gamma}{4} \int_{0}^{2 \pi} u_{2}(\omega) R\left(\xi+r_{1} e^{i \theta}, \xi+r_{2} e^{i \omega}\right) d \omega \\
& -l_{2}(u) \\
\mathcal{L}_{2,2}(u)(\theta)= & -\frac{\gamma}{4} \int_{0}^{2 \pi} u_{1}(\omega) R\left(\xi+r_{2} e^{i \theta}, \xi+r_{1} e^{i \omega}\right) d \omega \\
& +\frac{\gamma}{4} \int_{0}^{2 \pi} u_{2}(\omega) R\left(\xi+r_{2} e^{i \theta}, \xi+r_{2} e^{i \omega}\right) d \omega \\
& +\frac{\gamma u_{2}(\theta)}{4 r_{2}} \int_{R} \nabla R\left(\xi+r_{2} e^{i \theta}, y\right) \cdot e^{i \theta} d y \\
& +l_{2}(u),
\end{aligned}
$$

where $l_{2}(u)$ is real valued and is included so that $\mathcal{L}_{2}(u)$ is in $\mathcal{Y}$.

Because

$$
R\left(\xi+r_{k} e^{i \theta}, \xi+r_{l} e^{i \theta}\right)-R(\xi, \xi)=O\left(a^{1 / 2}\right)
$$

and $\int_{0}^{2 \pi} u_{k}(\omega) d \omega=0$, we obtain that

$$
\begin{aligned}
& \left\|\frac{\gamma}{4} \int_{0}^{2 \pi} u_{j}(\omega) R\left(\xi+r_{k} e^{i \theta}, \xi+r_{l} e^{i \omega}\right) d \omega\right\|_{L^{2}} \\
& \quad=\left\|\frac{\gamma}{4} \int_{0}^{2 \pi} u_{j}(\omega)\left(R\left(\xi+r_{k} e^{i \theta}, \xi+r_{l} e^{i \omega}\right)-R(\xi, \xi)\right) d \omega\right\|_{L^{2}} \\
& \quad \leq C \gamma a^{1 / 2}\left\|u_{j}\right\|_{L^{2}} \\
& \quad \leq C a^{-1}\left\|u_{j}\right\|_{L^{2}} .
\end{aligned}
$$


Since the area of $R$ is of order $O(a)$,

$$
\left\|\frac{\gamma u_{k}(\theta)}{4 r_{k}} \int_{R} \nabla R\left(\xi+r_{k} e^{i \theta}, y\right) \cdot e^{i \theta} d y\right\|_{L^{2}} \leq C a^{-1}\left\|u_{k}\right\|_{L^{2}} .
$$

The condition

$$
\overline{-\mathcal{L}_{2,1}(u)+\mathcal{L}_{2,2}(u)}=0
$$

in the definition of $\mathcal{Y}$ implies that

$$
\left|l_{2}(u)\right| \leq C a^{-1}\|u\|_{L^{2}} .
$$

The lemma then follows.

Lemma 5.3 1. For $u \in \mathcal{X}_{*},\|u\|_{H^{2}} \leq C a^{3 / 2}\|\Pi \mathcal{L}(u)\|_{L^{2}}$.

2. If $\left(S_{0,1}, \Gamma\right)$ lies below all the $W_{n}$ 's, then $\|u\|_{H^{1}}^{2} \leq C a^{3 / 2}\langle\Pi \mathcal{L}(u), u\rangle$.

3. $\Pi \mathcal{L}: \mathcal{X}_{*} \rightarrow \mathcal{Y}_{*}$ is one-to-one and onto.

Proof. When $a$ is small, by Lemma 5.1 Part 1 and Lemma 5.2,

$$
\|\Pi \mathcal{L}(u)\|_{L^{2}} \geq\left\|\Pi \mathcal{L}_{1}(u)\right\|_{L^{2}}-\left\|\Pi \mathcal{L}_{2}(u)\right\|_{L^{2}} \geq C a^{-3 / 2}\|u\|_{L^{2}}-C a^{-1}\|u\|_{L^{2}} \geq C a^{-3 / 2}\|u\|_{L^{2}},
$$

proving Part 1 of the lemma.

With condition 1 of Theorem 2.2, it follows from Lemma 5.1 Part 2 and Lemma 5.2 that

$$
\langle\Pi \mathcal{L}(u), u\rangle=\left\langle\Pi \mathcal{L}_{1}(u), u\right\rangle+\left\langle\Pi \mathcal{L}_{2}(u), u\right\rangle \geq C a^{-3 / 2}\|u\|_{H^{1}}^{2}-C a^{-1}\|u\|_{L^{2}}^{2} \geq C a^{-3 / 2}\|u\|_{H^{1}}^{2}
$$

when $a$ is sufficiently small.

Part 1 of this lemma ensures that $\Pi \mathcal{L}$ is one-to-one from $\mathcal{X}_{*}$ to $\mathcal{Y}_{*}$. Since $\Pi \mathcal{L}$ is self-adjoint and hence closed, it also ensures that the range of $\Pi \mathcal{L}$ is closed. The Closed Range Theorem (See Yosida [29, Page 205], e.g.) then implies that $\Pi \mathcal{L}$ is onto.

Finally in this section we state a bound on the second Fréchet derivative of $\mathcal{S}=\mathcal{H}+\mathcal{A}+\mathcal{B}+\lambda$.

Lemma 5.4 Assume that $\|\phi\|_{H^{2}} \leq c a$ where $c$ is sufficiently small. The following estimates hold for $u=\left(u_{1}, u_{2}\right) \in \mathcal{X}, v=\left(v_{1}, v_{2}\right) \in \mathcal{X}$.

1. $\left\|\mathcal{H}_{k}^{\prime \prime}\left(\phi_{k}\right)\left(u_{k}, v_{k}\right)\right\|_{L^{2}} \leq C a^{-5 / 2}\left\|u_{k}\right\|_{H^{2}}\left\|v_{k}\right\|_{H^{2}}$.

2. $\left\|\mathcal{A}^{\prime \prime}(\phi)(u, v)\right\|_{L^{2}} \leq C a^{-5 / 2}\|u\|_{H^{1}}\|v\|_{H^{1}}$.

3. $\left\|\mathcal{B}^{\prime \prime}(\phi)(u, v)\right\|_{L^{2}} \leq C a^{-2}\|u\|_{H^{1}}\|v\|_{H^{1}}$.

4. $\left|\lambda^{\prime \prime}(\phi)(u, v)\right| \leq C a^{-5 / 2}\|u\|_{H^{2}}\|v\|_{H^{2}}$.

In summary $\left\|\mathcal{S}^{\prime \prime}(\phi)(u, v)\right\|_{L^{2}} \leq C a^{-5 / 2}\|u\|_{H^{2}}\|v\|_{H^{2}}$.

Note that by taking $c$ small, we keep $r_{k}^{2}+\phi_{k}$ positive, so $E_{\phi}$ is a perturbed ring. The proof of this lemma is similar to that of [24, Lemma 3.2] or that of [23, Lemma 6.1]. We omit the details. 


\section{Reduction to three dimensions}

In this section it will be proved that, for each $\xi$ and $r_{1}$, there exists a pair of functions $\varphi\left(\cdot, \xi, r_{1}\right)=$ $\left(\varphi_{1}\left(\cdot, \xi, r_{1}\right), \varphi_{2}\left(\cdot, \xi, r_{1}\right)\right) \in \mathcal{X}_{*}$ such that

$$
\mathcal{S}(\varphi)(\theta)=A_{1} \cos \theta\left[\begin{array}{l}
r \\
1
\end{array}\right]+A_{2} \sin \theta\left[\begin{array}{l}
r \\
1
\end{array}\right]+\left[\begin{array}{l}
B_{1} \\
B_{2}
\end{array}\right]
$$

for some real numbers $A_{1}, A_{2}, B_{1}, B_{2}$. Note that $\varphi$ is sought in $\mathcal{X}_{*}$.

The equation (6.1) is written as

$$
\Pi \mathcal{S}\left(\varphi\left(\cdot, \xi, r_{1}\right)\right)=0
$$

where $\Pi$ is the orthogonal projection operator from $\mathcal{Y}$ to $\mathcal{Y}_{*}$. In the next section we will find a particular $\xi$ and $r_{1}$, say $\zeta$ and $s_{1}$, such that at $\xi=\zeta$ and $r_{1}=s_{1}, A_{1}=A_{2}=B_{1}=B_{2}=0$, i.e. $\mathcal{S}\left(\varphi\left(\cdot, \zeta, s_{1}\right)\right)=0$. This means that by finding $\varphi$ one reduces the original problem (1.1) to a problem of finding $\zeta$ and $s_{1}$ in a three dimensional set.

Recall $\mathcal{L}$, the linearized operator of $\mathcal{S}$ at $\phi=0$, i.e. $\mathcal{L}(u)=\mathcal{S}^{\prime}(0)(u)$. Expand $\mathcal{S}(\phi)$ as

$$
\mathcal{S}(\phi)=\mathcal{S}(0)+\mathcal{L}(\phi)+\mathcal{N}(\phi)
$$

where $\mathcal{N}$ is a higher order term defined by (6.3). Rewrite (6.2) in a fixed point form:

$$
\phi=-(\Pi \mathcal{L})^{-1}(\Pi \mathcal{S}(0)+\Pi \mathcal{N}(\phi))
$$

Lemma 6.1 There is $\varphi=\varphi\left(\cdot, \xi, r_{1}\right)$ such that for every $\xi \in U_{1}$ and $r_{1} \in U_{2}, \varphi\left(\cdot, \xi, r_{1}\right) \in \mathcal{X}_{*}$ solves (6.4) and $\left\|\varphi\left(\cdot, \xi, r_{1}\right)\right\|_{H^{2}} \leq c a^{3 / 2}$ where $c$ is a sufficiently large constant independent of $a$, $\xi$, and $r_{1}$.

Proof. To use the Contraction Mapping Principle in the fixed point setting (6.4), let

$$
\mathcal{T}(\phi)=-(\Pi \mathcal{L})^{-1}(\Pi \mathcal{S}(0)+\Pi \mathcal{N}(\phi))
$$

be an operator defined on

$$
D(\mathcal{T})=\left\{\phi \in \mathcal{X}_{*}:\|\phi\|_{H^{2}} \leq c a^{3 / 2}\right\}
$$

where the constant $c$ is sufficiently large and will be made more precise later.

We know from Lemma 3.1 that $\mathcal{S}(0)$ is a sum of a $\theta$ independent part and a quantity of order $O(1)$. After one applies $\Pi$ the $\theta$ independent part becomes 0 and we have

$$
\|\Pi \mathcal{S}(0)\|_{L^{2}}=O(1) .
$$

From Lemma 5.3 we deduce that

$$
\left\|(\Pi \mathcal{L})^{-1} \Pi \mathcal{S}(0)\right\|_{H^{2}} \leq C a^{3 / 2} .
$$

Lemma 5.4 implies that

$$
\|\mathcal{N}(\phi)\|_{L^{2}} \leq C a^{-5 / 2}\|\phi\|_{H^{2}}^{2}
$$

and consequently

$$
\left\|(\Pi \mathcal{L})^{-1} \Pi \mathcal{N}(\phi)\right\|_{H^{2}} \leq C a^{-1}\|\phi\|_{H^{2}}^{2} .
$$

Using (6.5), (6.8), (6.6), and (6.10) we find that

$$
\|\mathcal{T}(\phi)\|_{H^{2}} \leq C a^{3 / 2}+C a^{-1} c^{2} a^{3} \leq c a^{3 / 2}
$$

if $c$ is sufficiently large and $a$ sufficiently small. Therefore $\mathcal{T}$ is a map from $D(\mathcal{T})$ into itself. 
Finally we show that $\mathcal{T}$ is a contraction. Let $\phi_{1}, \phi_{2} \in D(\mathcal{T})$. First note that

$$
\mathcal{T}\left(\phi_{1}\right)-\mathcal{T}\left(\phi_{2}\right)=(\Pi \mathcal{L})^{-1}(-\Pi)\left(\mathcal{N}\left(\phi_{1}\right)-\mathcal{N}\left(\phi_{2}\right)\right)
$$

Because

$$
\mathcal{N}\left(\phi_{1}\right)-\mathcal{N}\left(\phi_{2}\right)=\mathcal{S}\left(\phi_{1}\right)-\mathcal{S}\left(\phi_{2}\right)-\mathcal{S}^{\prime}(0)\left(\phi_{1}-\phi_{2}\right),
$$

we deduce, with the help of Lemma 5.4 and (6.6), that

$$
\begin{aligned}
\left\|\mathcal{N}\left(\phi_{1}\right)-\mathcal{N}\left(\phi_{2}\right)\right\|_{L^{2}} & \leq\left\|\mathcal{S}^{\prime}\left(\phi_{2}\right)\left(\phi_{1}-\phi_{2}\right)-\mathcal{S}^{\prime}(0)\left(\phi_{1}-\phi_{2}\right)\right\|_{L^{2}}+C a^{-5 / 2}\left\|_{\phi_{1}}-\phi_{2}\right\|_{H^{2}}^{2} \\
& \leq C a^{-5 / 2}\left\|\phi_{2}\right\|_{H^{2}}\left\|\phi_{1}-\phi_{2}\right\|_{H^{2}}+C a^{-5 / 2}\left\|\phi_{1}-\phi_{2}\right\|_{H^{2}}^{2} \\
& \leq C a^{-5 / 2}\left(\left\|\phi_{1}\right\|_{H^{2}}+\left\|\phi_{2}\right\|_{H^{2}}\right)\left\|\phi_{1}-\phi_{2}\right\|_{H^{2}} \\
& \leq C a^{-1}\left\|\phi_{1}-\phi_{2}\right\|_{H^{2}} .
\end{aligned}
$$

Then Lemma 5.3 implies that

$$
\left\|\mathcal{T}\left(\phi_{1}\right)-\mathcal{T}\left(\phi_{2}\right)\right\|_{H^{2}} \leq C a^{1 / 2}\left\|\phi_{1}-\phi_{2}\right\|_{H^{2}}
$$

Therefore $\mathcal{T}$ is a contraction mapping in $D(\mathcal{T})$, if $a$ is sufficiently small. There is a fixed point $\varphi$, which we write as $\varphi=\varphi\left(\theta, \xi, r_{1}\right)$. Being in $D(\mathcal{T}),\|\varphi\|_{H^{2}}=O\left(a^{3 / 2}\right)$, which is smaller than the radius $r_{1}^{2}$ and $r_{2}^{2}$. Hence $E_{\varphi}$ is a perturbed ring.

We state a result regarding the linearization of $\mathcal{S}$ at $\varphi\left(\cdot, \xi, r_{1}\right)$. Denote this linearized operator by $\tilde{\mathcal{L}}$, i.e.

$$
\tilde{\mathcal{L}}=\mathcal{S}^{\prime}(\varphi)
$$

We have the following analogy of Lemma 5.3.

Lemma 6.2 1. There exists $C>0$ such that for all $u \in \mathcal{X}_{*}$

$$
\|u\|_{H^{2}} \leq C a^{3 / 2}\|\Pi \tilde{\mathcal{L}}(u)\|_{L^{2}}
$$

2. If the hypothesis of Theorem 2.2 holds, then

$$
\|u\|_{H^{1}}^{2} \leq C a^{3 / 2}\langle\Pi \tilde{\mathcal{L}}(u), u\rangle .
$$

Proof. By Lemma 5.3, Lemma 5.4, and the fact $\|\varphi\|_{H^{2}}=O\left(a^{3 / 2}\right)$, we deduce

$$
\begin{aligned}
\|\Pi \tilde{\mathcal{L}}(u)\|_{L^{2}} & \geq\|\Pi \mathcal{L}(u)\|_{L^{2}}-\|\Pi(\tilde{\mathcal{L}}-\mathcal{L})(u)\|_{L^{2}} \\
& \geq C a^{-3 / 2}\|u\|_{H^{2}}-C a^{-5 / 2}\|\varphi\|_{H^{2}}\|u\|_{H^{2}} \\
& \geq C a^{-3 / 2}\|u\|_{H^{2}}-C a^{-1}\|u\|_{H^{2}} \\
& \geq C a^{-3 / 2}\|u\|_{H^{2}}
\end{aligned}
$$

when $a$ is small.

If the hypothesis of Theorem 2.2 holds, then Lemmas 5.3 and 5.4 imply that

$$
\begin{aligned}
\langle\Pi \tilde{\mathcal{L}}(u), u\rangle & =\langle\Pi \mathcal{L}(u), u\rangle+\langle\Pi(\tilde{\mathcal{L}}-\mathcal{L}) u, u\rangle \\
& \geq C a^{-3 / 2}\|u\|_{H^{1}}^{2}-C a^{-5 / 3}\|\varphi\|_{H^{2}}\|u\|_{H^{1}}^{2} \\
& \geq C a^{-3 / 2}\|u\|_{H^{1}}^{2}-C a^{-1}\|u\|_{H^{1}}^{2} \\
& \geq C a^{-3 / 2}\|u\|_{H^{1}}^{2} .
\end{aligned}
$$

This proves the second part of the lemma.

One consequence of this lemma is an estimate of $\frac{\partial \varphi}{\partial \xi_{j}}$. 
Lemma $6.3 \varphi$ satisfies $\left\|\frac{\partial \varphi}{\partial \xi_{j}}\right\|_{H^{2}}=O(a), j=1,2$.

Proof. We prove this lemma by the Implicit Function Theorem. Differentiating $\Pi \mathcal{S}(\varphi)$ with respect to $\xi_{j}$ finds that

$$
\begin{aligned}
0 & =\frac{\partial \Pi \mathcal{S}_{k}(\varphi)}{\partial \xi_{j}} \\
& =\Pi \tilde{\mathcal{L}}_{k}\left(\frac{\partial \varphi}{\partial \xi_{j}}\right)+\Pi \gamma(-1)^{k} \int_{E_{\varphi}}\left[\frac{\partial R\left(\xi+\sqrt{r_{k}^{2}+\varphi_{k}(\theta)} e^{i \theta}, y\right)}{\partial x_{j}}+\frac{\partial R\left(\xi+\sqrt{r_{k}^{2}+\varphi_{k}(\theta)} e^{i \theta}, y\right)}{\partial y_{j}}\right] d y
\end{aligned}
$$

where $R=R(x, y)$. It is clear that

$$
\left\|\int_{E_{\varphi}}\left[\frac{\partial R\left(\xi+\sqrt{r_{k}^{2}+\varphi_{k}(\theta)} e^{i \theta}, y\right)}{\partial x_{j}}+\frac{\partial R\left(\xi+\sqrt{r_{k}^{2}+\varphi_{k}(\theta)} e^{i \theta}, y\right)}{\partial y_{j}}\right] d y\right\|_{L^{2}}=O(a) .
$$

With the help of Lemma 6.2 we deduce that

$$
\left\|\frac{\partial \varphi}{\partial \xi_{j}}\right\|_{H^{2}} \leq C a^{3 / 2} \gamma a=C a .
$$

\section{$7 \quad$ Existence and stability}

We prove Theorems 2.1 and 2.2 in this section. From Lemma 6.1 we know that for every $\xi \in U_{1}$ and $r_{1} \in U_{2}$ there exists $\varphi\left(\cdot, \xi, r_{1}\right) \in \mathcal{X}_{*}$ such that $\Pi \mathcal{S}\left(\varphi\left(\cdot, \xi, r_{1}\right)\right)=0$, i.e. (6.1) holds. In this section we find particular $\xi$ and $r_{1}$ denoted by $\zeta$ and $s_{1}$ such that $\mathcal{S}\left(\varphi\left(\cdot, \zeta, s_{1}\right)\right)=0$.

Lemma 7.1 $J\left(E_{\varphi\left(\cdot, \xi, r_{1}\right)}\right)=J(R)+O\left(a^{3 / 2}\right)$.

Proof. Expanding $J\left(E_{\varphi}\right)$ yields

$$
J\left(E_{\varphi}\right)=J(R)+\sum_{k=1}^{2} \int_{0}^{2 \pi} \mathcal{S}_{k}(0) \varphi_{k} d \theta+\frac{1}{2} \sum_{k=1}^{2} \int_{0}^{2 \pi} \mathcal{L}_{k}(\varphi) \varphi_{k} d \theta+O\left(a^{2}\right) .
$$

The error term in (7.1) is obtained by Lemma 5.4. and the fact $\|\varphi\|_{H^{2}}=O\left(a^{3 / 2}\right)$.

On the other hand $\Pi \mathcal{S}(\varphi)=0$ implies that

$$
\Pi(\mathcal{S}(0)+\mathcal{L}(\varphi)+\mathcal{N}(\varphi))=0
$$

where $\mathcal{N}$ is given in (6.3). We multiply the last equation by $\varphi$ and integrate to derive, again with the help of Lemma 5.4,

$$
\sum_{k=1}^{2} \int_{0}^{2 \pi} \mathcal{S}_{k}(0) \varphi_{k} d \theta+\sum_{k=1}^{2} \int_{0}^{2 \pi} \mathcal{L}_{k}(\varphi) \varphi_{k} d \theta=O\left(a^{2}\right)
$$

We can now rewrite (7.1) as

$$
J\left(E_{\varphi}\right)=J(R)+\frac{1}{2} \sum_{k=1}^{2} \int_{0}^{2 \pi} \mathcal{S}_{k}(0) \varphi_{k} d \theta+O\left(a^{2}\right) .
$$

Lemma 3.1 and the fact $\|\varphi\|_{H^{2}}=O\left(a^{3 / 2}\right)$ implies that

$$
J\left(E_{\varphi}\right)=J(R)+O\left(a^{3 / 2}\right)+O\left(a^{2}\right)=J(R)+O\left(a^{3 / 2}\right) .
$$

When we use Lemma 3.1, note that $\mathcal{S}(0)$ is a sum of a $\theta$ independent part and a quantity of order $O(1)$, and that $\varphi_{k} \perp 1$. This proves the lemma. 
Lemma 7.2 As a function of $\left(\xi, r_{1}\right), J\left(E_{\varphi\left(\cdot, \xi, r_{1}\right)}\right)$ is locally minimized at some $\left(\zeta, s_{1}\right)$, when a is small. As $a \rightarrow 0$,

$$
\zeta \rightarrow \zeta_{0}, \quad\left(\frac{a|D|}{\pi}\right)^{-1 / 2} s_{1} \rightarrow S_{0,1}
$$

possibly along a subsequence, where $R\left(\zeta_{0}, \zeta_{0}\right)=\min _{x \in D} R(x, x)$.

Proof. If we consider $J\left(\varphi\left(\cdot, \xi, r_{1}\right)\right)$ as a function of $\xi$ and $r_{1}$, then Lemmas 3.2 and 7.1 imply that

$$
\begin{aligned}
J\left(E_{\varphi}\right)= & 2 \pi\left(r_{1}+r_{2}\right)+\frac{\gamma}{2}\left[\frac{\pi}{2} r_{1}^{4} \log \frac{1}{r_{1}}+\frac{\pi}{2} r_{2}^{4} \log \frac{1}{r_{2}}-\pi r_{1}^{2} r_{2}^{2} \log \frac{1}{r_{2}}-\frac{a|D|}{4} r_{1}^{2}\right. \\
& \left.+a^{2}|D|^{2} R(\xi, \xi)+\frac{a^{2}|D|^{2}}{8 \pi}+\frac{a^{2}|D|}{4}\left(r_{1}^{2}+r_{2}^{2}\right)\right]+O\left(a^{3 / 2}\right) \\
= & 2 \pi\left(\frac{a|D|}{\pi}\right)^{1 / 2}\left\{\left(R_{1}+\sqrt{1+R_{1}^{2}}\right)+\frac{\Gamma}{4}\left[-\frac{1}{2} \log \left(\frac{a|D|}{\pi}\right)^{1 / 2}+\frac{1}{8}+\pi R(\xi, \xi)\right.\right. \\
& \left.\left.+\frac{R_{1}^{2}}{4}\left(R_{1}^{2} \log \frac{1+R_{1}^{2}}{R_{1}^{2}}-1\right)+\frac{1}{4} \log \frac{1}{1+R_{1}^{2}}+\frac{a}{4}\left(2 R_{1}^{2}+1\right)\right]\right\}+O\left(a^{3 / 2}\right) .
\end{aligned}
$$

Here we have used the scaled variables $R_{j}$ and $\Gamma$ where

$$
R_{j}=\left(\frac{a|D|}{\pi}\right)^{-1 / 2} r_{j}, j=1,2 ; \quad \Gamma=\left(\frac{a|D|}{\pi}\right)^{3 / 2} \gamma,
$$

with the new constraint $R_{2}^{2}-R_{1}^{2}=1$. Denote the leading order, $R_{1}$ dependent part by

$$
Q_{\Gamma}\left(R_{1}\right)=R_{1}+\sqrt{1+R_{1}^{2}}+\frac{\Gamma}{4}\left[\frac{R_{1}^{2}}{4}\left(R_{1}^{2} \log \frac{1+R_{1}^{2}}{R_{1}^{2}}-1\right)+\frac{1}{4} \log \frac{1}{1+R_{1}^{2}}\right]
$$

so that

$$
J\left(E_{\varphi\left(\cdot, \xi, r_{1}\right)}\right)=2 \pi\left(\frac{a|D|}{\pi}\right)^{1 / 2}\left\{Q_{\Gamma}\left(R_{1}\right)+\frac{\Gamma}{4}\left[-\frac{1}{2} \log \left(\frac{a|D|}{\pi}\right)^{1 / 2}+\frac{1}{8}+\pi R(\xi, \xi)\right]\right\}+O\left(a^{3 / 2}\right) .
$$

By our assumption that $S_{0,1}$ locally minimizes $Q_{\Gamma}$, the lemma is proved.

We show that $\varphi\left(\cdot, \zeta, s_{1}\right)$ is an exact solution of (1.1) in the next two lemmas.

Lemma 7.3 At $\xi=\zeta$ and $r_{1}=s_{1}$,

$$
\mathcal{S}(\varphi)=A_{1} \cos \theta\left[\begin{array}{l}
r \\
1
\end{array}\right]+A_{2} \sin \theta\left[\begin{array}{l}
r \\
1
\end{array}\right] .
$$

In other words at $\left(\zeta, s_{1}\right)$,

$$
\left[\begin{array}{l}
B_{1} \\
B_{2}
\end{array}\right]=\left[\begin{array}{l}
0 \\
0
\end{array}\right]
$$

Proof. Let

$$
p=\left(p_{1}, p_{2}\right), q=\left(q_{1}, q_{2}\right) \text { where } p_{k}=r_{k}^{2}, q_{k}=s_{k}^{2}, k=1,2 .
$$

In this proof we view $J\left(E_{\varphi}\right)$ as a function of $\xi$ and $p$. Calculations show that

$$
\begin{aligned}
\frac{\partial J\left(E_{\varphi}\right)}{\partial p_{k}} & =\sum_{l=1}^{2} \int_{0}^{2 \pi}\left[\mathcal{H}_{l}(\varphi)+\mathcal{A}_{l}(\varphi)+\mathcal{B}_{l}(\varphi)\right] \frac{\partial\left(p_{l}+\varphi_{l}\right)}{\partial p_{k}} \\
& =\sum_{l=1}^{2} \int_{0}^{2 \pi}\left[\mathcal{S}_{l}(\varphi)-(-1)^{l} \lambda(\varphi)\right]\left(\delta_{l k}+\frac{\partial \varphi_{l}}{\partial p_{k}}\right) d \theta \\
& =\int_{0}^{2 \pi}\left[\mathcal{S}_{k}(\varphi)-(-1)^{k} \lambda(\varphi)\right] d \theta+\sum_{l=1}^{2} \int_{0}^{2 \pi}\left[\mathcal{S}_{l}(\varphi)-(-1)^{l} \lambda(\varphi)\right] \frac{\partial \varphi_{l}}{\partial p_{k}} d \theta \\
& =2 \pi B_{k}-2 \pi(-1)^{k} \lambda(\varphi) .
\end{aligned}
$$


Here $\delta_{l k}=1$ if $l=k$ and $=0$ otherwise. We have also used the fact that $\frac{\partial \varphi}{p_{k}} \in \mathcal{X}_{*} \subset \mathcal{Y}_{*}$, which follows from $\varphi \in \mathcal{X}_{*}$, and the fact that $\mathcal{S}(\varphi) \perp \mathcal{Y}_{*}$.

On the other hand at the minimum $p=q$ and $\xi=\zeta$, we must have

$$
\left.\frac{\partial J\left(E_{\varphi}\right)}{\partial p_{k}}\right|_{\xi=\zeta, p=q}=(-1)^{k} \mu, \quad k=1,2 \text {. }
$$

Here $\mu$ is a Lagrange multiplier coming from the constraint $p_{2}-p_{1}=\frac{a|D|}{\pi}$. Therefore at $\zeta$ and $q$,

$$
2 \pi B_{1}+2 \pi \lambda=-\mu, 2 \pi B_{2}-2 \pi \lambda=\mu
$$

which imply that

$$
B_{1}+B_{2}=0 .
$$

According to the definition of $\mathcal{S}, \int_{0}^{2 \pi}\left(-\mathcal{S}_{1}(\varphi)+\mathcal{S}_{2}(\varphi)\right) d \theta=0$ as in (4.16), which implies that

$$
-B_{1}+B_{2}=0
$$

We deduce from (7.4) and (7.5) that $B_{1}=B_{2}=0$.

We prove the existence of a solution in the next lemma. It uses a tricky re-parametrization technique.

Lemma 7.4 At $\xi=\zeta$ and $r_{1}=s_{1}, \mathcal{S}(\varphi)=0$.

Proof. For $\xi=\left(\xi_{1}, \xi_{2}\right)$ near $\zeta$ we re-parametrize $\partial_{D} E_{\varphi\left(\cdot, \xi, r_{1}\right)}$. Let $\zeta$ be the center of a new polar coordinates, $r_{1}^{2}+\psi_{1}, r_{2}^{2}+\psi_{2}$ the new inner and outer radii square and $\eta$ the new angle. A point on $\partial_{D} E_{\varphi\left(\cdot, \xi, r_{1}\right)}$ is described as $\zeta+\sqrt{r_{1}^{2}+\psi_{1}} e^{i \eta}$ or $\zeta+\sqrt{r_{2}^{2}+\psi_{2}} e^{i \eta}$. It is related to the old polar coordinates via

$$
\zeta+\sqrt{r_{k}^{2}+\psi_{k}} e^{i \eta}=\xi+\sqrt{r_{k}^{2}+\varphi_{k}} e^{i \theta}
$$

for $k=1,2$.

In the new coordinates $E_{\varphi}$ becomes $E_{\psi}$. It is viewed as a perturbation of the ring centered at $\zeta$ with radii $r_{1}, r_{2}$. The perturbation is described by $\psi=\left(\psi_{1}, \psi_{2}\right)$ which is a pair of functions of $\eta$. It also depends on $\xi$ and $r$.

The main effect of the new coordinates is to "freeze" the center. The center of the new polar system is $\zeta$ which is fixed while the center of the old polar system is $\xi$ which varies in $U$.

We now consider the derivative of $J\left(E_{\varphi\left(\cdot, \xi, r_{1}\right)}\right)=J\left(E_{\psi\left(\cdot, \xi, r_{1}\right)}\right)$ with respect to $\xi$. On one hand, at $\xi=\zeta$ and $r=s$,

$$
\left.\frac{\partial J\left(E_{\psi\left(\cdot, \xi, r_{1}\right)}\right)}{\partial \xi_{j}}\right|_{\xi=\zeta, r=s}=\left.\frac{\partial J\left(E_{\varphi\left(\cdot, \xi, r_{1}\right)}\right)}{\partial \xi_{j}}\right|_{\xi=\zeta, r=s}=0, \quad j=1,2,
$$

since $(\zeta, s)$ is a minimum.

On the other hand calculations show that

$$
\frac{\partial J\left(E_{\psi\left(\cdot, \xi, r_{1}\right)}\right)}{\partial \xi_{j}}=\sum_{l=1}^{2} \int_{0}^{2 \pi} \mathcal{S}_{l}(\psi(\cdot, \xi))(\eta) \frac{\partial \psi_{l}}{\partial \xi_{j}} d \eta
$$

We emphasize that (7.8) is obtained under the re-parametrized coordinates, in which the dependence of $J\left(E_{\psi\left(\cdot, \xi, r_{1}\right)}\right)$ on $\xi$ is only reflected in the dependence of $\psi$ on $\xi$. Had we calculated in the original coordinates, $\xi$ would have appeared also in the nonlocal part of $J$ through $R(\xi+\ldots, \xi+\ldots)$. The result would have been very different from (7.8). See the proof of Lemma 6.3 which involves differentiation 
with respect to $\xi$ in the original coordinates. In the derivation of (7.8) we have used the fact that $\int_{0}^{2 \pi} \psi_{l} d \eta=0$ which implies that $\int_{0}^{2 \pi} \frac{\partial \psi_{l}}{\partial \xi_{j}} d \eta=0$, so that $\int_{0}^{2 \pi} \lambda(\psi) \frac{\partial \psi_{l}}{\partial \xi_{j}} d \eta=0$ where $\lambda(\psi)$ is part of

$$
\mathcal{S}_{l}(\psi)=\mathcal{H}_{l}(\psi)+\mathcal{A}_{l}(\psi)+\mathcal{B}_{l}(\psi)+(-1)^{l} \lambda(\psi)
$$

and we can reach the right side of (7.8).

The expression $\mathcal{S}(\phi)$ is invariant under re-parametrization, i.e.

$$
\mathcal{S}\left(\varphi\left(\cdot, \xi, r_{1}\right)\right)(\theta)=\mathcal{S}\left(\psi\left(\cdot, \xi, r_{1}\right)\right)(\eta) .
$$

Now we return to the original coordinate system and integrate with respect to $\theta$ in (7.8). Then

$$
\frac{\partial J\left(E_{\psi\left(\cdot, \xi, r_{1}\right)}\right)}{\partial \xi_{j}}=\sum_{l=1}^{2} \int_{0}^{2 \pi} \mathcal{S}_{l}(\varphi)(\theta) \frac{\partial \psi_{l}(\eta(\theta, \xi), \xi)}{\partial \xi_{j}} \frac{\partial \eta}{\partial \theta} d \theta
$$

We recall that $\psi$ and $\eta$ are defined implicitly as functions of $\theta$ and $\xi$ by (7.6). Let us agree that $\psi_{k}=\psi_{k}(\eta, \xi)$ is a function of $\eta$ and $\xi$. Set $\Psi_{k}(\theta, \xi)=\psi_{k}(\eta(\theta, \xi), \xi)$. Implicit differentiation shows that, with the help of Lemmas 6.1 and 6.3,

$$
\begin{aligned}
& {\left[\begin{array}{lll}
\frac{\partial \eta}{\partial \theta} & \frac{\partial \eta}{\partial \xi_{1}} & \frac{\partial \eta}{\partial \xi_{2}} \\
\frac{\partial \Psi_{k}}{\partial \theta} & \frac{\partial \Psi_{k}}{\partial \xi_{1}} & \frac{\partial \Psi_{k}}{\partial \xi_{2}}
\end{array}\right]=-\left[\begin{array}{cc}
\sqrt{r_{k}^{2}+\Psi_{k}} \sin \eta & -\frac{\cos \eta}{2 \sqrt{r_{k}^{2}+\Psi_{k}}} \\
-\sqrt{r_{k}^{2}+\Psi_{k}} \cos \eta & -\frac{\sin \eta}{2 \sqrt{r_{k}^{2}+\Psi_{k}}}
\end{array}\right]} \\
& \quad \times \quad\left[\begin{array}{lll}
\frac{\cos \theta}{2 \sqrt{r_{k}^{2}+\varphi_{k}}} \frac{\partial \varphi_{k}}{\partial \theta}-\sqrt{r_{k}^{2}+\varphi_{k}} \sin \theta & 1+\frac{\cos \theta}{2 \sqrt{r_{k}^{2}+\varphi_{k}}} \frac{\partial \varphi_{k}}{\partial \xi_{1}} & \frac{\cos \theta}{2 \sqrt{r_{k}^{2}+\varphi_{k}}} \frac{\partial \varphi_{k}}{\partial \xi_{2}} \\
\frac{\sin \theta}{2 \sqrt{r_{k}^{2}+\varphi_{k}}} \frac{\partial \varphi_{k}}{\partial \theta}+\sqrt{r_{k}^{2}+\varphi_{k}} \cos \theta & \frac{\sin \theta}{2 \sqrt{r_{k}^{2}+\varphi_{k}}} \frac{\partial \varphi_{k}}{\partial \xi_{1}} & 1+\frac{\sin \theta}{2 \sqrt{r_{k}^{2}+\varphi_{k}}} \frac{\partial \varphi_{k}}{\partial \xi_{2}}
\end{array}\right] \\
& =2\left[\begin{array}{lll}
\frac{-\sin \eta}{2 \sqrt{r_{k}^{2}+\Psi_{k}}} & \frac{\cos \eta}{2 \sqrt{r_{k}^{2}+\Psi_{k}}} \\
\sqrt{r_{k}^{2}+\Psi_{k}} \cos \eta & \sqrt{r_{k}^{2}+\Psi_{k}} \sin \eta
\end{array}\right] \\
& \times \quad\left[\begin{array}{lll}
-\sqrt{r_{k}^{2}+\varphi_{k}} \sin \theta+O(a) & 1+O\left(a^{1 / 2}\right) & O\left(a^{1 / 2}\right) \\
\sqrt{r_{k}^{2}+\varphi_{k}} \cos \theta+O(a) & O\left(a^{1 / 2}\right) & 1+O\left(a^{1 / 2}\right)
\end{array}\right] .
\end{aligned}
$$

At $\xi=\zeta$ and $r=s, \eta=\theta$ and $\Psi_{k}=\varphi_{k}$ and the above becomes

$$
\left[\begin{array}{lll}
\frac{\partial \eta}{\partial \theta} & \frac{\partial \eta}{\partial \xi_{1}} & \frac{\partial \eta}{\partial \xi_{2}} \\
\frac{\partial \Psi_{k}}{\partial \theta} & \frac{\partial \Psi_{k}}{\partial \xi_{1}} & \frac{\partial \Psi_{k}}{\partial \xi_{2}}
\end{array}\right]=\left[\begin{array}{lll}
1+O\left(a^{1 / 2}\right) & -\frac{\sin \theta}{\sqrt{r_{k}^{2}+\varphi_{k}}}+O(1) & \frac{\cos \theta}{\sqrt{r_{k}^{2}+\varphi_{k}}}+O(1) \\
O\left(a^{3 / 2}\right) & 2 \sqrt{r_{k}^{2}+\varphi_{k}} \cos \theta+O(a) & 2 \sqrt{r_{k}^{2}+\varphi_{k}} \sin \theta+O(a)
\end{array}\right]
$$

We have found that at $\xi=\zeta$ and $r=s$,

$$
\frac{\partial \Psi_{k}}{\partial \xi_{1}}=2 r_{k} \cos \theta+O(a), \quad \frac{\partial \Psi_{k}}{\partial \xi_{2}}=2 r_{k} \sin \theta+O(a) .
$$

To compute $\frac{\partial \psi_{k}}{\partial \xi_{j}}$, we invert $\eta=\eta(\xi, \theta)$ to express $\theta=\Theta(\eta, \xi)$. Then

$$
\frac{\partial \psi_{k}}{\partial \xi_{j}}=\frac{\partial \Psi_{k}}{\partial \xi_{j}}+\frac{\partial \Psi_{k}}{\partial \theta} \frac{\partial \Theta}{\partial \xi_{j}}
$$

At $\xi=\zeta$ and $r=s$, since

$$
\frac{\partial \Psi_{k}}{\partial \theta}=O\left(a^{3 / 2}\right), \quad \frac{\partial \Theta}{\partial \xi_{j}}=-\frac{\frac{\partial \eta}{\partial \xi_{j}}}{\frac{\partial \eta}{\partial \theta}}=O\left(a^{-1 / 2}\right)
$$


we deduce that

$$
\frac{\partial \psi_{k}}{\partial \xi_{1}}=2 r_{k} \cos \theta+O(a), \quad \frac{\partial \psi_{k}}{\partial \xi_{2}}=2 r_{k} \sin \theta+O(a)
$$

Following (7.14) and the fact that $\frac{\partial \eta}{\partial \theta}=1+O\left(a^{1 / 2}\right)$ we find that at $\xi=\zeta$ and $r=s,(7.10)$ becomes

$$
\begin{aligned}
& \frac{\partial J\left(E_{\psi}\right)}{\partial \xi_{1}}=\sum_{l=1}^{2} \int_{0}^{2 \pi} \mathcal{S}_{l}(\varphi)\left(2 r_{l} \cos \theta+O(a)\right) d \theta \\
& \frac{\partial J\left(E_{\psi}\right)}{\partial \xi_{2}}=\sum_{l=1}^{2} \int_{0}^{2 \pi} \mathcal{S}_{l}(\varphi)\left(2 r_{l} \sin \theta+O(a)\right) d \theta .
\end{aligned}
$$

Now we combine (6.1), (7.7) and (7.15) to derive that

$$
\begin{aligned}
& A_{1} \int_{0}^{2 \pi}\left[\left(r r_{1}+r_{2}\right) \cos ^{2} \theta+O(a)\right] d \theta+A_{2} \int_{0}^{2 \pi}\left[\left(r r_{1}+r_{2}\right) \cos \theta \sin \theta+O(a)\right] d \theta=0 \\
& A_{1} \int_{0}^{2 \pi}\left[\left(r r_{1}+r_{2}\right) \cos \theta \sin \theta+O(a)\right] d \theta+A_{2} \int_{0}^{2 \pi}\left[\left(r r_{1}+r_{2}\right) \sin ^{2} \theta+O(a)\right] d \theta=0 .
\end{aligned}
$$

Writing the system in the matrix form

$$
\left(\left[\begin{array}{ll}
\pi\left(r r_{1}+r_{2}\right) & 0 \\
0 & \pi\left(r r_{1}+r_{2}\right)
\end{array}\right]+O(a)\right)\left[\begin{array}{l}
A_{1} \\
A_{2}
\end{array}\right]=\left[\begin{array}{l}
0 \\
0
\end{array}\right],
$$

since (7.16) is non-singular when $a$ is small, we deduce that $A_{1}=A_{2}=0$, proving the lemma.

We know now that $\varphi\left(\cdot, \zeta, s_{1}\right)$ found in Lemma 7.2 solves $\mathcal{S}(\phi)=0$ and hence the equation (1.1). The center of the perturbed ring solution $E_{\varphi\left(\cdot, \zeta, s_{1}\right)}$ is $\zeta$, the inner radius is $s_{1}$ and the outer radius is $s_{2}=\sqrt{\frac{a|D|}{\pi}+s_{1}^{2}}$. As $a \rightarrow 0, \zeta \rightarrow \zeta_{0}$ and $\left(\frac{a|D|}{\pi}\right)^{-1 / 2} s_{1} \rightarrow S_{0,1}$ possibly along a subsequence, where $\zeta_{0}$ minimizes the function $R(x, x)$ in $D$. This proves Theorem 2.1.

In Theorem 2.2, a solution is termed stable if it is a local minimizer of $J$ in the space

$$
\left(U_{1} \times U_{2}\right) \times\left\{\phi=\left(\phi_{1}, \phi_{2}\right): \phi_{k} \in H^{1}\left(S^{1}\right), k=1,2, \phi \in \mathcal{Y}_{*}\right\} .
$$

In Theorem 2.2 if $\left(S_{0,1}, \Gamma\right)$ lies below all the $W_{n}$ 's, Lemma 6.2, Part 2 , shows that each $\varphi(\cdot, \xi, r)$ we found in Lemma 6.1 locally minimizes $J$, with fixed $(\xi, r) \in U_{1} \times U_{2}$, in $\left\{\phi: \phi_{k} \in H^{1}\left(S^{1}\right), \phi \in \mathcal{Y}_{*}\right\}$. On the other hand $\varphi\left(\cdot, \zeta, s_{1}\right)$ minimizes $J\left(E_{\varphi\left(\cdot, \xi, r_{1}\right)}\right)$ with respect to $\left(\xi, r_{1}\right)$ in $U_{1} \times U_{2}$. Hence $\varphi\left(\cdot, \zeta, s_{1}\right)$ is a local minimizer of $J$ in $(7.17)$.

If $\left(S_{0,1}, \Gamma\right)$ lies between two curves, there is $n \in\{2,3, \ldots\}$ such that $\left(S_{0,1}, \Gamma\right)$ is above the curve $W_{n}$. Then the eigenvalue $\lambda_{n, 2}$ of $\mathcal{L}_{1}$ is negative. There exists $C>0$ such that

$$
\lambda_{n, 2}<-C a^{-3 / 2}, \quad\left\langle\mathcal{L}_{1}\left(e_{n, 2}\right), e_{n, 2}\right\rangle<-C a^{-3 / 2}\left\|e_{n, 2}\right\|_{L^{2}}^{2}
$$

where $e_{n, 2}$ is an eigenvector of $\mathcal{L}_{1}$ corresponding to $\lambda_{n, 2}$. By Lemma 5.2, the last inequality implies that

$$
\left\langle\mathcal{L}\left(e_{n, 2}\right), e_{n, 2}\right\rangle<-C a^{-3 / 2}\left\|e_{n, 2}\right\|_{L^{2}}^{2} .
$$

Then by Lemma 5.4

$$
\left\langle\tilde{\mathcal{L}}\left(e_{n, 2}\right), e_{n, 2}\right\rangle<-C a^{-3 / 2}\left\|e_{n, 2}\right\|_{L^{2}}^{2} .
$$

Therefore the solution is unstable. This proves Theorem 2.2. 


\section{Discussion}

In Figure 4 the graph of $Q_{\Gamma}$ shows that when $\Gamma$ is large, $Q_{\Gamma}$ also has a local maximum in addition to the local minimum $S_{0,1}$. This local maximum indicates the existence of an unstable ring pattern solution whose inner radius corresponds to the local maximum on the graph of $Q_{\Gamma}$.

To prove this assertion one uses the same argument and reduces the problem to $J\left(E_{\varphi\left(\cdot, \xi, r_{1}\right)}\right)$. However instead of Lemma 7.2 where a local minimum of $J\left(E_{\varphi\left(\cdot, \xi, r_{1}\right)}\right)$ is found, we have to prove the existence of a saddle point for $J\left(E_{\varphi\left(\cdot, \xi, r_{1}\right)}\right)$. Roughly speaking we would like to minimize $J\left(E_{\varphi\left(\cdot, \xi, r_{1}\right)}\right)$ with respect to $\xi$ and maximize $J\left(E_{\varphi\left(\cdot, \xi, r_{1}\right)}\right)$ with respect to $r_{1}$. This intuitive idea may be made rigorous by a type of mini-max argument.

This unstable solution is probably a mountain-pass type saddle point between the ring solution found in this paper and a single droplet solution found by Ren and Wei in [24]. A single droplet solution of (1.1) is a set $E$ which is close to a small disc. Under the setting of the current paper, the main result of [24] may be stated as follows.

Theorem 8.1 ([24]) For any compact subset $\mathcal{K}$ of $(0, \infty) \backslash\{2 n(n+1): n=2,3,4, \ldots\}$, there exists a constant $a_{0}>0$ such that if

$$
a<a_{0} \text { and } \gamma\left(\frac{a|D|}{\pi}\right)^{3 / 2} \in \mathcal{K}
$$

there is a droplet solution of (1.1). The radius of the droplet is close to $\left(\frac{a|D|}{\pi}\right)^{1 / 2}$, and the center of the droplet is close to a minimum of $R(x, x), x \in D$.

If $\gamma\left(\frac{a|D|}{\pi}\right)^{3 / 2}<2 n(n+1)$ for all $n=2,3, \ldots$ (in other words $\left.\gamma\left(\frac{a|D|}{\pi}\right)^{3 / 2}<12\right)$, then the solution is stable; if $\gamma\left(\frac{a|D|}{\pi}\right)^{3 / 2}>2 n(n+1)$ for some $n \in\{2,3, \ldots\}$, then the solution is unstable.

Another interesting feature of the ring solution found in Observation 2.3 is that for the ring solution to be stable $\gamma$ must be large, i.e.

$$
\gamma\left(\frac{a|D|}{\pi}\right)^{3 / 2}>\Gamma_{1}
$$

But for a droplet solution to be stable, according to Theorem 8.1, $\gamma$ must be small, i.e.

$$
\gamma\left(\frac{a|D|}{\pi}\right)^{3 / 2}<12
$$

According to Figure $3, \Gamma_{1}>12$. We can not have a stable ring and a stable droplet for the same $\gamma$.

\section{A Appendix}

In this appendix we show that

$$
\begin{aligned}
& \int_{\tilde{R}} \frac{\left(r_{1} e^{i \theta}-y\right) \cdot e^{i \theta}}{\left|r_{1} e^{i \theta}-y\right|^{2}} d y=0 \\
& \int_{\tilde{R}} \frac{\left(r_{2} e^{i \theta}-y\right) \cdot e^{i \theta}}{\left|r_{2} e^{i \theta}-y\right|^{2}} d y=\pi\left(r_{2}-\frac{r_{1}^{2}}{r_{2}}\right)
\end{aligned}
$$

where $\tilde{R}=B_{r_{2}}(0) \backslash B_{r_{1}}(0), B_{r_{1}}(0)$ is the disc centered at 0 with radius $r_{1}$, and $B_{r_{2}}(0)$ is the disc centered at 0 with radius $r_{2}$.

We first calculate the integral

$$
\int_{B_{r_{1}}(0)} \frac{\left(r_{1} e^{i \theta}-y\right) \cdot e^{i \theta}}{\left|r_{1} e^{i \theta}-y\right|^{2}} d y
$$


Scale $B_{r_{1}}(0)$ to $B_{1}(0)$, the disc centered at 0 of radius 1 , so that

$$
\int_{B_{r_{1}}(0)} \frac{\left(r_{1} e^{i \theta}-y\right) \cdot e^{i \theta}}{\left|r_{1} e^{i \theta}-y\right|^{2}} d y=r_{1} \int_{B_{1}(0)} \frac{\left(e^{i \theta}-y\right) \cdot e^{i \theta}}{\left|e^{i \theta}-y\right|^{2}} d y
$$

Let $y=e^{i \theta}((1,0)-z)$, and $z=r e^{i \beta}$. The disc $B_{1}(0)$ now becomes $B_{1}(1,0)$, the disc centered at $(1,0)$ of radius 1 . Its boundary is parametrized in the polar coordinates by $r=2 \cos \beta$. Then we have

$$
\int_{B_{1}(0)} \frac{\left(e^{i \theta}-y\right) \cdot e^{i \theta}}{\left|e^{i \theta}-y\right|^{2}} d y=\int_{B_{1}(1,0)} \frac{e^{i \theta} z \cdot e^{i \theta}}{|z|^{2}} d z=\int_{-\pi / 2}^{\pi / 2} \int_{0}^{2 \cos \beta} \cos \beta d r d \beta=\pi .
$$

We have our first formula

$$
\int_{B_{r_{1}}(0)} \frac{\left(r_{1} e^{i \theta}-y\right) \cdot e^{i \theta}}{\left|r_{1} e^{i \theta}-y\right|^{2}} d y=\pi r_{1}
$$

Next we calculate

$$
\int_{B_{r_{2}}(0)} \frac{\left(r_{1} e^{i \theta}-y\right) \cdot e^{i \theta}}{\left|r_{1} e^{i \theta}-y\right|^{2}} d y=r_{1} \int_{B_{1 / r}(0)} \frac{\left(e^{i \theta}-y\right) \cdot e^{i \theta}}{\left|e^{i \theta}-y\right|^{2}} d y
$$

Here $r=r_{1} / r_{2}<1$. Let $y=e^{i \theta}((1,0)-z)$, then using the polar coordinates, we turn the above integral to

$$
\begin{aligned}
r_{1} \int_{B_{1 / r}(0)} \frac{\left(e^{i \theta}-y\right) \cdot e^{i \theta}}{\left|e^{i \theta}-y\right|^{2}} d y & =r_{1} \int_{B_{1 / r}(1,0)} \frac{z_{1}}{|z|^{2}} d z \\
& =r_{1} \int_{0}^{2 \pi} \int_{0}^{\cos \beta+\sqrt{\frac{1}{r^{2}}-\sin ^{2} \beta}} \cos \beta d \rho d \beta \\
& =r_{1} \int_{0}^{2 \pi} \cos \beta\left(\cos \beta+\sqrt{\left.\frac{1}{r^{2}}-\sin ^{2} \beta\right)} d \beta\right. \\
& =\pi r_{1} .
\end{aligned}
$$

We have our second formula

$$
\int_{B_{r_{2}}(0)} \frac{\left(r_{1} e^{i \theta}-y\right) \cdot e^{i \theta}}{\left|r_{1} e^{i \theta}-y\right|^{2}} d y=\pi r_{1} .
$$

(A.1) follows from (A.3) and (A.4).

Similarly to (A.3) we have

$$
\int_{B_{r_{2}}(0)} \frac{\left(r_{2} e^{i \theta}-y\right) \cdot e^{i \theta}}{\left|r_{2} e^{i \theta}-y\right|^{2}} d y=\pi r_{2}
$$

But different from (A.4) we see that

$$
\int_{B_{r_{1}}(0)} \frac{\left(r_{2} e^{i \theta}-y\right) \cdot e^{i \theta}}{\left|r_{2} e^{i \theta}-y\right|^{2}} d y=r_{2} \int_{B_{r}(0)} \frac{\left(e^{i \theta}-y\right) \cdot e^{i \theta}}{\left|e^{i \theta}-y\right|^{2}} d y
$$

and the function

$$
y \rightarrow \frac{\left(e^{i \theta}-y\right) \cdot e^{i \theta}}{\left|e^{i \theta}-y\right|^{2}}
$$

is harmonic (without singularity) for $y \in B_{r}(0)$. The Mean Value Theorem for harmonic functions implies that

$$
\int_{B_{r}(0)} \frac{\left(e^{i \theta}-y\right) \cdot e^{i \theta}}{\left|e^{i \theta}-y\right|^{2}} d y=\pi r^{2}
$$


We now have our last formula

$$
\int_{B_{r_{1}}(0)} \frac{\left(r_{2} e^{i \theta}-y\right) \cdot e^{i \theta}}{\left|r_{2} e^{i \theta}-y\right|^{2}} d y=\pi \frac{r_{1}^{2}}{r_{2}} .
$$

(A.2) follows from (A.5) and (A.6).

\section{B Appendix}

To show (5.12) for $r<1$, we expand

$$
\log (1-z)=-\sum_{n=1}^{\infty} \frac{z^{n}}{n}
$$

for $z \in C,|z|<1$.

Let $z=r e^{i \theta}$, and notice that $\log \left|1-r e^{i \theta}\right|$ is just the real part of the analytic function $\log (1-z)$ in the unit disc. Equating the real parts on both sides of (B.1) gives the desired result.

\section{References}

[1] F. S. Bates and G. H. Fredrickson. Block copolymers - designer soft materials. Physics Today, $52(2): 32-38,1999$.

[2] X. Chen and Y. Oshita. Periodicity and uniqueness of global minimizers of an energy functional containing a long-range interaction. SIAM J. Math. Anal., 37(4):1299-1332, 2005.

[3] R. Choksi. Scaling laws in microphase separation of diblock copolymers. J. Nonlinear Sci., 11(3):223-236, 2001.

[4] R. Choksi and X. Ren. On the derivation of a density functional theory for microphase separation of diblock copolymers. J. Statist. Phys., 113(1-2):151-176, 2003.

[5] R. Choksi and X. Ren. Diblock copolymer - homopolymer blends: derivation of a density functional theory. Physica D, 203(1-2):100-119, 2005.

[6] R. Choksi and P. Sternberg. On the first and second variations of a nonlocal isoperimetric problem. J. Reine Angew. Math., 2007(611):75-108, 2007.

[7] L.C. Evans and R.F. Gariepy. Measure Theory and Fine Properties of Functions. CRC Press, Boca Raton, New York, London, Tokyo, 1992.

[8] P. C. Fife and D. Hilhorst. The Nishiura-Ohnishi free boundary problem in the 1D case. SIAM J. Math. Anal., 33(3):589-606, 2001.

[9] S. Müller. Singular perturbations as a selection criterion for periodic minimizing sequences. Calc. Var. Partial Differential Equations, 1(2):169-204, 1993.

[10] Y. Nishiura and I. Ohnishi. Some mathematical aspects of the microphase separation in diblock copolymers. Physica D, 84(1-2):31-39, 1995.

[11] I. Ohnishi, Y. Nishiura, M. Imai, and Y. Matsushita. Analytical solutions describing the phase separation driven by a free energy functional containing a long-range interaction term. Chaos, $9(2): 329-341,1999$. 
[12] T. Ohta and K. Kawasaki. Equilibrium morphology of block copolymer melts. Macromolecules, 19(10):2621-2632, 1986.

[13] X. Ren and J. Wei. On the multiplicity of solutions of two nonlocal variational problems. SIAM J. Math. Anal., 31(4):909-924, 2000.

[14] X. Ren and J. Wei. Concentrically layered energy equilibria of the di-block copolymer problem. European J. Appl. Math., 13(5):479-496, 2002.

[15] X. Ren and J. Wei. On energy minimizers of the di-block copolymer problem. Interfaces Free Bound., 5(2):193-238, 2003.

[16] X. Ren and J. Wei. On the spectra of 3-D lamellar solutions of the diblock copolymer problem. SIAM J. Math. Anal., 35(1):1-32, 2003.

[17] X. Ren and J. Wei. Triblock copolymer theory: Free energy, disordered phase and weak segregation. Physica D, 178(1-2):103-117, 2003.

[18] X. Ren and J. Wei. Triblock copolymer theory: Ordered ABC lamellar phase. J. Nonlinear Sci., 13(2):175-208, 2003.

[19] X. Ren and J. Wei. Stability of spot and ring solutions of the diblock copolymer equation. $J$. Math. Phys., 45(11):4106-4133, 2004.

[20] X. Ren and J. Wei. Wriggled lamellar solutions and their stability in the diblock copolymer problem. SIAM J. Math. Anal., 37(2):455-489, 2005.

[21] X. Ren and J. Wei. Droplet solutions in the diblock copolymer problem with skewed monomer composition. Calc. Var. Partial Differential Equations, 25(3):333-359, 2006.

[22] X. Ren and J. Wei. Existence and stability of spherically layered solutions of the diblock copolymer equation. SIAM J. Appl. Math., 66(3):1080-1099, 2006.

[23] X. Ren and J. Wei. Many droplet pattern in the cylindrical phase of diblock copolymer morphology. Rev. Math. Phys., 19(8):879-921, 2007.

[24] X. Ren and J. Wei. Single droplet pattern in the cylindrical phase of diblock copolymer morphology. J. Nonlinear Sci., 17(5):471-503, 2007.

[25] X. Ren and J. Wei. Spherical solutions to a nonlocal free boundary problem from diblock copolymer morphology. SIAM J. Math. Anal., 39(5):1497-1535, 2008.

[26] T. Teramoto and Y. Nishiura. Double gyroid morphology in a gradient system with nonlocal effects. Journal of the Physical Society of Japan, 71(7):1611-1614, 2002.

[27] G. P. Tolstov. Fourier Series. Prentice-Hall, INC, Englewood Cliffs, New Jersey, 1962.

[28] Y. Tsori, D. Andelman, and M. Schick. Defects in lamellar diblock copolymers: Chevron- and $\Omega$-shaped boundaries. Phys. Rev. E, 61(3):2848-2858, 2000.

[29] K. Yosida. Functional Analysis. Springer-Verlag, Berlin Heidelberg New York, 1980. 